\title{
Genotyping-by-sequencing and SNP-arrays are complementary for detecting quantitative trait loci by tagging different haplotypes in association studies
}

Sandra S. Negro ${ }^{1}$, Emilie J. Millet ${ }^{2,3}$, Delphine Madur ${ }^{1}$, Cyril Bauland ${ }^{1}$, Valérie Combes ${ }^{1}$, Claude Welcker², François Tardieu ${ }^{2}$, Alain Charcosset ${ }^{1}$ and Stéphane D. Nicolas ${ }^{1 *}$

\begin{abstract}
Background: Single Nucleotide Polymorphism (SNP) array and re-sequencing technologies have different properties (e.g. calling rate, minor allele frequency profile) and drawbacks (e.g. ascertainment bias). This lead us to study their complementarity and the consequences of using them separately or combined in diversity analyses and Genome-Wide Association Studies (GWAS). We performed GWAS on three traits (grain yield, plant height and male flowering time) measured in 22 environments on a panel of 247 F1 hybrids obtained by crossing 247 diverse dent maize inbred lines with a same flint line. The 247 lines were genotyped using three genotyping technologies (Genotyping-By-Sequencing, Illumina Infinium $50 \mathrm{~K}$ and Affymetrix Axiom $600 \mathrm{~K}$ arrays).

Results: The effects of ascertainment bias of the $50 \mathrm{~K}$ and $600 \mathrm{~K}$ arrays were negligible for deciphering global genetic trends of diversity and for estimating relatedness in this panel. We developed an original approach based on linkage disequilibrium (LD) extent in order to determine whether SNPs significantly associated with a trait and that are physically linked should be considered as a single Quantitative Trait Locus (QTL) or several independent QTLs. Using this approach, we showed that the combination of the three technologies, which have different SNP distributions and densities, allowed us to detect more QTLs (gain in power) and potentially refine the localization of the causal polymorphisms (gain in resolution).

Conclusions: Conceptually different technologies are complementary for detecting QTLs by tagging different haplotypes in association studies. Considering LD, marker density and the combination of different technologies (SNP-arrays and re-sequencing), the genotypic data available were most likely enough to well represent polymorphisms in the centromeric regions, whereas using more markers would be beneficial for telomeric regions.
\end{abstract}

Keywords: GWAS, Linkage disequilibrium, Genome coverage, Maize, High-throughput genotyping technologies

\section{Background}

Understanding the genetic bases of complex traits involved in the adaptation to biotic and abiotic stress in plants is a pressing concern, with world-wide drought due to climate change as a major source of human food and agriculture threats. Recent progress in next generation sequencing and genotyping array technologies

\footnotetext{
* Correspondence: stephane.nicolas@inra.fr

${ }^{1} \mathrm{GQE}$ - Le Moulon, INRA, Univ. Paris-Sud, CNRS, AgroParisTech, Université

Paris-Saclay, 91190 Gif-sur-Yvette, France

Full list of author information is available at the end of the article
}

contribute to a better understanding of the genetic basis of quantitative trait variation by allowing Genome-Wide Association Studies (GWAS) on large diversity panels [1]. Single Nucleotide Polymorphism (SNP)-based techniques became the most commonly used genotyping methods for GWAS because SNPs are cheap, numerous, codominant and can be automatically analysed with SNP-arrays or produced by genotyping-by-sequencing (GBS), or sequencing [2-4]. The decreasing cost of genotyping technologies has led to an exponential increase in the number of markers used for the GWAS in

(c) The Author(s). 2019 Open Access This article is distributed under the terms of the Creative Commons Attribution 4.0 International License (http://creativecommons.org/licenses/by/4.0/), which permits unrestricted use, distribution, and 
association panels, thereby raising the question of computation time to perform the association tests. Computational issues were addressed by using either approximate methods by avoiding re-estimating variance components for each SNP [5] or exact methods using mathematical tools for sparing time in matrix inversion [6, 7]. It is noteworthy that using approximate computation in GWAS can produce inaccurate $p$-values when the SNP effect size is large or/and when the sample structure is strong [8].

Several causes may impact the power of Quantitative Trait Locus (QTL, locus involved in quantitative trait variation) detection in GWAS. Highly diverse panels have in general undergone multiple historical recombination, leading to a low extent of linkage disequilibrium (LD). However, these panels can present different average and local patterns of LD [9-11]. A high marker density and a proper distribution of SNPs are therefore essential to capture causal polymorphisms. Furthermore, minor allele frequencies (MAF), population stratification and cryptic relatedness are three other important parameters affecting power and false positive detection [12, 13]. These last two factors are substantial in several cultivated species such as maize [14] and grapevine [15], and their impact on LD can be statistically evaluated [16]. Population structure and kinship can be estimated using molecular markers [17-20] and can be modelled to efficiently detect marker-trait associations due to linkage only $[12,21,22]$. These advances have largely increased the power and effectiveness of linear mixed models that can now efficiently account for population structure and relatedness in GWAS $[8,12]$.

In maize, an Illumina Infinium HD 50,000 SNP-array, named MaizeSNP50 array (hereafter $50 \mathrm{~K}$ ) was developed by Ganal et al. [3] and has been used extensively for diversity and association studies [23, 24]. For example, GWAS were conducted to unravel the genetic architecture of phenology, yield component traits and to identify several flowering time QTLs linked to adaptation of tropical maize to temperate climate $[25,26]$. In the same way, Rincent et al. [11] showed that LD occurs over a longer distance in a dent than in a flint panel, with appreciable effects on the power of QTL detection. Low LD extent and relationship between allelic frequencies with population and pedigree structure at some SNPs reduce the power of GWAS $[14,26]$. Therefore, higher marker densities are desirable because the maize genome size is large $(2.4 \mathrm{~Gb})$, the level of diversity is high (more than one substitution per hundred nucleotides), and LD extent is low [27]. As a consequence, an Affymetrix Axiom 600,000 SNP-array (hereafter $600 \mathrm{~K}$ ) was developed and used in association genetics $[28,29]$ and detection of selective sweeps [4]. Another possibility is whole genome sequencing, but this is currently impractical for large genomes such as maize because of the associated cost. Hence, a Genotyping-By-Sequencing (GBS) procedure has been developed [2] that targets low-copy genomic regions by using restriction enzymes. Genotyping-by-sequencing technology is cost-effective and has been successfully used in maize for genomic prediction [30]. Romay et al. [31] and Gouesnard et al. [32] highlighted the interest of the GBS for (i) deciphering and comparing the genetic diversity of the inbred lines in seedbanks and (ii) identifying QTLs by GWAS for kernel colour, sweet corn and flowering time.

Few studies in plants have compared datasets from different high-throughput genotyping technologies [33-35]. Elbasyoni et al. [32] used GBS and a $90 \mathrm{~K}$ SNP-array in winter wheat. They highlighted strong positive correlations between the population structure matrices and kinships identified by both technologies. They showed that GBSSNPs led to higher genomic prediction accuracy compared to Array-SNPs. Torkamaneh and Belzile [35] used GBS and a $50 \mathrm{~K}$ SNP-array in soybean. They estimated ca. $98 \%$ accuracy of genotype called by their GBS pipeline and showed that the accuracy of imputation for missing genotypes was hardly affected by the chosen MAF and only moderately affected by the rate of missing values. Li et al. [34] created a reliable integrated variation map using a $600 \mathrm{~K}$ and $50 \mathrm{~K}$ SNP-array, GBS and RNA sequencing to dissect regulatory causality and its link to maize kernel variation. These authors used a fixed physical distance $(<10 \mathrm{~kb})$ for grouping associated SNPs into QTLs despite the variable LD pattern along the genome. None of these studies compared QTL detection between the different technologies.

The main drawback of the DNA arrays is that they do not allow the discovery of new SNPs. This possibly leads to some ascertainment bias in diversity analyses when the SNPs selected for building arrays come from (i) the sequencing of a set of individuals that did not represent well the diversity explored in the studied panel, (ii) a subset of SNPs that skews the allelic frequency profile towards the intermediate frequencies [27, 36]. Ascertainment bias can compromise the ability of the SNP-arrays to reveal an exact view of the genetic diversity [36]. Genotyping-by-sequencing can overcome ascertainment bias since it is based on sequencing and therefore allows the discovery of alleles in the diversity panel analysed. It can be generalized to any species at a low cost providing that numerous individuals have been sequenced in order to build a representative library of short haplotypes to call SNPs [37]. Non-repetitive regions of genomes can be targeted with two- to three-fold higher efficiency, thereby considerably reducing the computationally challenging problems associated with alignment in species with high repeat content. However, GBS may have low coverage leading to a high missing data rate $(65 \%$ in both studies; [32, 33]) and heterozygote under-calling, 
depending on genome size and structure, and on the multiplexing level per sequencing flow-cell. Furthermore, GBS requires the establishment of demanding bioinformatic pipelines and imputation algorithms [37]. Pipelines have been developed to call SNP genotypes from raw GBS sequence data and to impute the missing data from a haplotype library $[37,38]$.

Here, we investigated the impact of using GBS and SNP-arrays on the quality of the genotyping data, together with the biological properties of data generated by each technology, and the potential complementarity of these approaches. In particular, we analyzed the impact of marker density and genotyping technologies (sequencing vs array) on (i) the estimates of relatedness and population structure, and (ii) the detection of QTLs (power). To address these issues, we performed a GWAS based on genotypic datasets obtained using either GBS or SNP-arrays with low $(50 \mathrm{k})$ or high $(600 \mathrm{k})$ densities on a diversity panel of maize hybrids obtained by crossing a panel of dent lines with a common flint tester line. Three traits were considered, namely grain yield, plant height and male flowering time (day to anthesis), measured in 22 different environments (sites $\times$ years $\times$ treatments) over Europe. We developed an original approach based on LD extent in order to determine whether SNPs significantly associated with a trait should be considered as a single QTL or several independent QTLs.

\section{Results}

Combining TASSEL and beagle imputations improved the genotyping quality for GBS

We estimated the genotyping and imputation concordance of the GBS based on common markers with the 50 $\mathrm{K}$ or $600 \mathrm{~K}$ arrays (Additional file 1: Figure S1 and Table 1). The genotyping concordance of the $600 \mathrm{~K}$ with the $50 \mathrm{~K}$ was extremely high $(99.50 \%)$, although slightly lower for residual heterozygotes (92.88\%). After SNP calling from sequencing reads using AllZeaGBSv2.7 database (direct reads, $\mathrm{GBS}_{1}$, Additional file 1: Figure S1), the call rate was $33.81 \%$ for the common SNPs with the $50 \mathrm{~K}$, vs $37 \%$ for the whole GBS dataset. The genotyping concordance rate between the direct reads of GBS and the 50 K was $98.88 \%$ (Table 1). After imputation using TASSEL by Cornell Institute $\left(\mathrm{GBS}_{2}\right)$, the concordance rate was $96.04 \%$ on the common markers with the $50 \mathrm{~K}$ and $11.91 \%$ of missing data remained for the whole GBS dataset. In $\mathrm{GBS}_{3}$, all missing data were imputed by Beagle and the remaining missing genotypes in $\mathrm{GBS}_{2}$ were excluded here to be comparable with TASSEL. This method yielded a lower concordance rate (93.04 and $92.84 \%$ with the $50 \mathrm{~K}$ and $600 \mathrm{~K}$, respectively). In an attempt to increase the concordance rate of the genotyping while removing missing data, we tested two additional methods, namely $\mathrm{GBS}_{4}$ where the missing data and heterozygotes of Cornell imputed data $\left(\mathrm{GBS}_{2}\right)$ were replaced by Beagle imputation, and $\mathrm{GBS}_{5}$ where Cornell homozygous genotypes $\left(\mathrm{GBS}_{2}\right)$ were completed by imputations from $\mathrm{GBS}_{3}$ (Additional file 1: Figure $\mathrm{S} 1$ and Table 1$). \mathrm{GBS}_{5}$ displayed a slightly better concordance rate than $\mathrm{GBS}_{2}(96.25 \%$ vs $96.04 \%)$ and predicted heterozygotes with a higher quality than $\mathrm{GBS}_{4} \cdot \mathrm{GBS}_{5}$ was therefore used for all genetic analyses and named GBS hereafter.

\section{GBS displayed rarer alleles and lower call rate than SNP- arrays}

The SNP call rate was higher for the SNP-arrays (average values of 96 and $>99 \%$ for the $50 \mathrm{~K}$ and $600 \mathrm{~K}$, respectively), than for the GBS ( $37 \%$ for the direct reads). The MAF distribution differed between the technologies (Additional file 2: Figure S2): while the use of SNP-arrays resulted in a near-uniform distribution, GBS resulted in an excess of rare alleles with a L-shaped distribution $(22 \%$ of SNPs with MAF $<0.05$ for the GBS versus 6 and $9 \%$ for the $50 \mathrm{~K}$ and $600 \mathrm{~K}$, respectively). This can be

Table 1 Percentage of GBS concordance and call rates (in parentheses)

\begin{tabular}{|c|c|c|c|c|}
\hline & Reference & Total & Homozygotes & Heterozygotes \\
\hline \multirow[t]{2}{*}{ GBS, Direct Read } & $50 \mathrm{~K}$ & $98.88(33.81)$ & $99.03(33.72)$ & $45.09(0.09)$ \\
\hline & $600 K$ & 98.99 (35.58) & $99.21(35.47)$ & $28.67(0.11)$ \\
\hline \multirow[t]{2}{*}{$\mathrm{GBS}_{2}$ Cornell Imputation } & $50 \mathrm{~K}$ & $96.04(91.56)$ & $98.66(88.79)$ & $12.51(2.78)$ \\
\hline & $600 \mathrm{~K}$ & $95.50(93.41)$ & $98.69(90.14)$ & $7.75(3.28)$ \\
\hline \multirow[t]{2}{*}{$\mathrm{GBS}_{3}$ Beagle Imputation } & $50 \mathrm{~K}$ & $93.04\left({ }^{\mathrm{a}} 91.56\right)$ & $93.23(91.30)$ & $30.54(0.26)$ \\
\hline & $600 \mathrm{~K}$ & $92.84\left({ }^{\mathrm{a}} 93.41\right)$ & 93.07 (93.12) & $22.50(0.29)$ \\
\hline \multirow{2}{*}{$\begin{array}{l}\mathrm{GBS}_{4} \text { Beagle Imputation on the missing data and heterozygotes } \\
\text { after TASSEL Imputation (GBS2) }\end{array}$} & $50 \mathrm{~K}$ & $96.46\left({ }^{a} 97.64\right)$ & $96.46(97.63)$ & $<0.01(<0.01)$ \\
\hline & $600 \mathrm{~K}$ & $96.21\left({ }^{\mathrm{a}} 99,97\right)$ & $96.21(>99.99)$ & $<0.01(<0.01)$ \\
\hline \multirow{2}{*}{$\begin{array}{l}\mathrm{GBS}_{5} \text { Compilation of Homoz. genotypes from TASSEL Imputation } \\
\text { (GBS2) and Imputation by Beagle for Other Data (GBS3) }\end{array}$} & $50 \mathrm{~K}$ & $96.25\left({ }^{\mathrm{a}} 97.65\right)$ & $96.36(97.47)$ & $39.07(0.18)$ \\
\hline & $600 \mathrm{~K}$ & $95.98\left({ }^{\mathrm{a} 99.97)}\right.$ & $96.11(99.78)$ & $32.02(0.22)$ \\
\hline
\end{tabular}

The $50 \mathrm{~K}$ and $600 \mathrm{~K}$ SNP-arrays were considered as reference genotypes. ${ }^{a}$ After Beagle inference of missing data, the call rate was $100 \%$. Here the call rate is $<$ $100 \%$ because the comparison was made against the $50 \mathrm{~K}$ and the $600 \mathrm{~K}$ that include few missing data. For $\mathrm{GBS}_{3}$, the remaining missing genotypes in $\mathrm{GBS}_{2}$ were also excluded to obtain comparable results 
explained by the fact that the $50 \mathrm{~K}$ was based on 27 sequenced lines for SNPs discovery [3], the $600 \mathrm{~K}$ was based on 30 lines [4], whereas GBS was based on 31, 978 lines, thereby leading to higher discovery of rare alleles. Consistent with MAF distribution, the average gene diversity $(\mathrm{He})$ was lower for GBS (0.27) than for arrays $(0.35$ and 0.34 for the $50 \mathrm{~K}$ and $600 \mathrm{~K}$ arrays, respectively). The distribution of SNP residual heterozygosity of inbred lines was similar for the three technologies, with a mean of $0.80,0.89$ and $0.22 \%$ for the $50 \mathrm{~K}, 600 \mathrm{~K}$ and GBS, respectively. The residual heterozygosity of inbred lines was highly correlated between technologies with large coefficients of Spearman correlation: $r_{50 \mathrm{~K}-600 \mathrm{~K}}=0.90, \mathrm{r}_{50 \mathrm{~K}-\mathrm{GBS}}=0.76$, $\mathrm{r}_{600 \mathrm{~K}-\mathrm{GBS}}=0.83$. The distribution of the SNPs along the genome was denser in the telomeres for the GBS and in the peri-centromeric regions for the $600 \mathrm{~K}$, whereas the $50 \mathrm{~K}$ exhibited a more uniform distribution (Fig. 1 and top graph in Additional file 3: Figure S3).

\section{Population structure and relatedness were consistent between the three technologies}

We used the ADMIXTURE software to analyse the genetic structure within the studied panel based on SNPs from the three technologies, by considering two to ten groups. Based on a K-fold cross-validation, the clustering in four genetic groups $\left(N_{Q}=4\right)$ was identified as the best for the three datasets. Considering a threshold of 0.5 for ancestral fraction, the assignation to the four groups was identical except for a few admixed inbred lines (Additional file 4: Figure S4). Based on the $50 \mathrm{~K}$, the four groups were constituted by (i) 39 lines in the Non Stiff Stalk (Iodent) family traced by PH207, (ii) 46 lines in the Lancaster family traced by Mo17 and Oh43, and (iii) 55 lines in the Stiff Stalk family traced by B73 and (iv) 107 lines that did not fit into these three primary heterotic groups, such as W117 and F7057. This organization appeared consistent with the organization of breeding programs into heterotic groups, generally related to few key founder lines.

We compared two estimators of relatedness between inbred lines, IBS (Identity-By-State) and $K \_$Freq (Identity-By-Descent), calculated per technology. For IBS, pairs of individuals were on average more related using GBS than SNP-arrays (mean IBS: 0.66, 0.67 and 0.73 for 50 $\mathrm{K}, 600 \mathrm{~K}$ and GBS, respectively). As expected, mean IBD was close for the three technologies (K_Freq: -0.004$)$. Relatedness estimates with the two SNP-arrays were highly correlated: $\mathrm{r}=0.95$ and 0.98 for IBS and $K_{-}$ Freq, respectively (Additional file 5: Figure S5b and d). Likewise, relatedness estimates between arrays and GBS were strongly correlated (between 0.94 and 0.98 , Additional file 5: Figure S5b and d).
We further carried out diversity analyses by performing Principal Coordinate Analyses (PCoA) on IBD ( $K_{-}$ Freq, weights by allelic frequency) estimated from the three technologies (Fig. 2). The three first PCoA axes explained 12.9, 15.6 and $16.3 \%$ of the variability for the GBS, $50 \mathrm{~K}$ and $600 \mathrm{~K}$, respectively (Fig. 2). The same pattern was observed regardless of the technology with the first axis separating the Stiff Stalk from all other groups (Iodent and Lancaster lines, see illustration with the 50 K kinship, Fig. 2). Key founder lines of the three heterotic groups (Iodent: PH207, Stiff Stalk: B73, Lancaster: Mo17) were found at extreme positions along the axes, which was consistent with the admixture groups previously described.

\section{Long distance linkage disequilibrium was removed by taking into account population structure or relatedness} In order to evaluate the effect of kinship and the genetic structure on linkage disequilibrium (LD), we studied genome-wide LD between 29,257 PANZEA markers from the $50 \mathrm{~K}$ within and between chromosomes before and after taking into account the kinship (K_Freq estimated from the $50 \mathrm{~K}$ ), structure (Number of groups $=4$ ) or both (Additional file 6: Figure S6). Whereas interchromosomal LD was only partially removed when the genetic structure was taken into account, it was mostly removed when either the kinship or both kinship and structure were considered (Additional file 6: Figure S6b and c). Accordingly, long distance intra-chromosomal LD was almost totally removed for all chromosomes by accounting for the kinship, structure or both. Interestingly, some pairs of loci located on different chromosomes or very distant on a same chromosome remained in high LD despite correction for genetic structure and kinship (Additional file 6: Figure S6). This can be explained either by genome assembly errors, by chromosomal rearrangements such as translocations or by strong epistatic interactions. Linkage disequilibrium decreased with genetic or physical distance (Fig. 3). The majority of pairs of loci with high $\operatorname{LD}\left(r^{2} K>0.4\right)$ in spite of long physical distance ( $>30 \mathrm{Mbp}$ ), were close genetically $(<3 \mathrm{cM})$, notably on chromosome $3,5,7$ and to a lesser extent 9 and 10 (data not shown). These loci were located in centromeric and peri-centromeric regions that displayed low recombination rate, suggesting that this pattern was due to variation of recombination rate along the chromosome. Only very few pairs of loci in high LD were genetically distant $(>5 \mathrm{cM})$ but physically close $(<2 \mathrm{Mbp})$. Linkage disequilibrium $\left(r^{2} K\right.$ and $\left.r^{2} K S\right)$ was negligible beyond $1 \mathrm{cM}$ since $99 \%$ of LD values were less than 0.12 in this case. Note that some unplaced SNPs remained in LD after taking into account the kinship and structure with some SNPs with known positions on chromosome 1, 3 and 4 (Additional file 6: Figure S6). Therefore, LD 


\section{Chromosome 3}

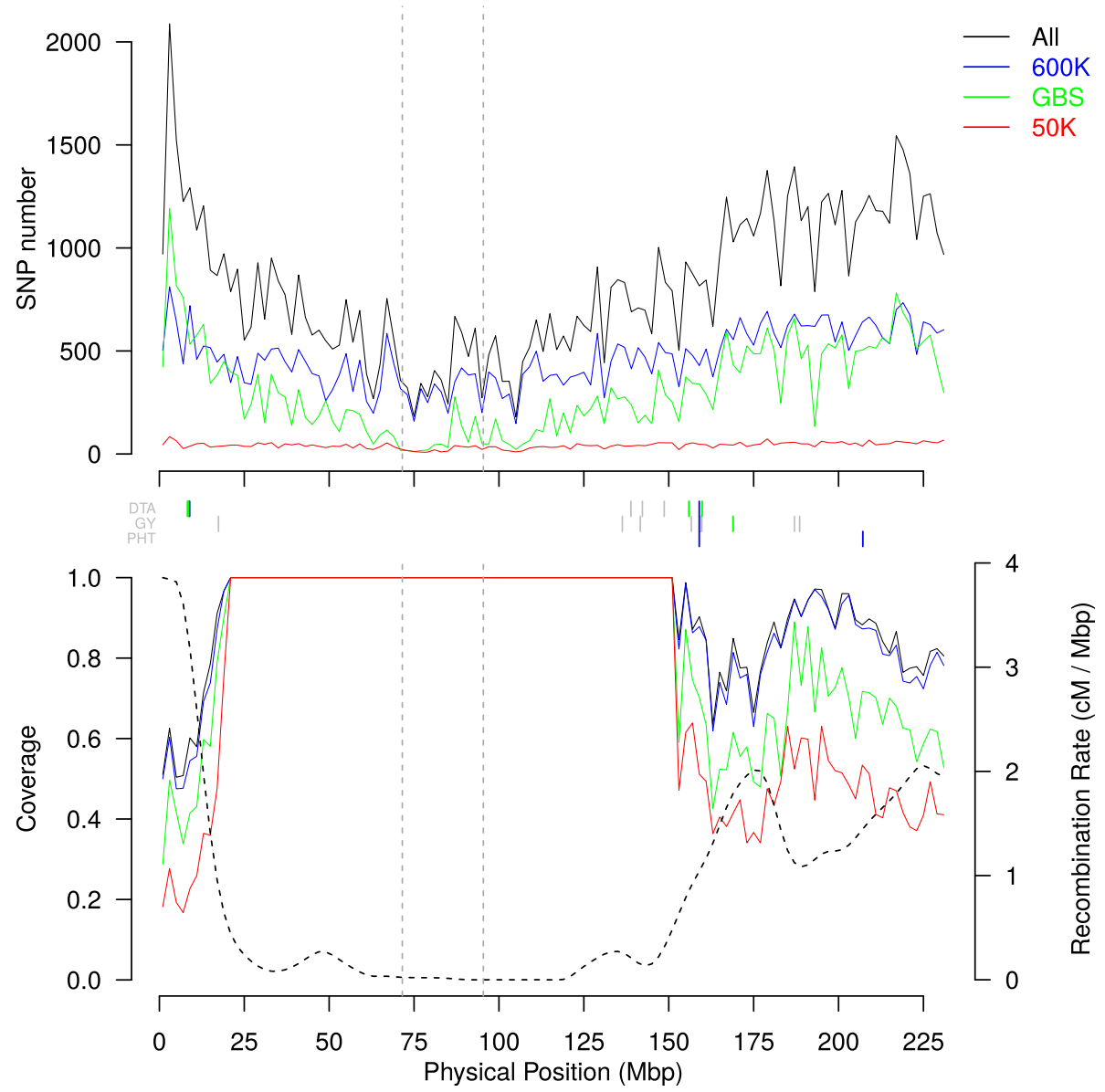

Fig. 1 Variation of the markers density, the recombination rate and the genome coverage in non-overlapping 2 Mbp windows along chromosome 3. Markers have MAF above 5\%. Top panel shows the variation of SNP number. In the bottom panel, dotted line represents the variation of recombination rate (cM / Mbp) and solid lines the proportion of genome covered by the SNPs using the cumulated length of physical LD windows around each SNP in each 2Mbp-windows. In these two panels, green, blue, red and black lines represent variation for GBS, $600 \mathrm{~K}, 50 \mathrm{~K}$ and combined technologies, respectively. Vertical dotted gray lines indicate limits of centromeric regions. Vertical lines between the two panels indicate the position of QTLs for flowering time (DTA), grain yield (GY) and Plant Height (PHT). Green, blue, red vertical lines indicate QTLs detected only by GBS, $600 \mathrm{~K}$ and $50 \mathrm{~K}$ technologies, respectively. Grey lines indicated QTLs detected by at least two technologies. Only QTLs including a marker associated with - $\log _{10}($ pval) above 6 were shown

measurement corrected by the kinship can help to map unplaced SNPs.

\section{Linkage disequilibrium strongly differed between and within chromosomes}

We combined the three technologies together to calculate the $r^{2} K$ for all pairs of SNPs which were genetically distant by less than $1 \mathrm{cM}$. For any chromosome region, LD extent in terms of genetic and physical distance showed a limited variation over the 100 sets of 500,000 loci pairs (cf. Material). This suggests that the estimation of LD extent did not strongly depend on our set of loci. LD extent varied significantly between chromosomes for both high recombinogenic $(>0.5 \mathrm{cM} / \mathrm{Mbp})$ and low recombinogenic regions ( $<0.5 \mathrm{cM} / \mathrm{Mbp}$, Table 2). Chromosome 1 had the highest
LD extent in high recombination regions $(0.062 \pm 0.007$ $\mathrm{cM}$ ) and chromosome 9 the highest LD extent in low recombinogenic regions ( $898.6 \pm 21.7 \mathrm{kbp})$ (Table 2). Linkage disequilibrium extent relative to genetic and physical distances was highly and positively correlated in high recombinogenic regions $(r=0.86)$, whereas it was not in low recombinogenic regions $(r=-0.64)$.

\section{Large differences in genome coverage between technologies}

We estimated the percentage of the genome that was covered by LD windows around SNPs, calculated by using either physical or genetic distances (Fig. 3, Table 2). We observed a strong difference in coverage between the three technologies at both genome-wide and chromosome scale, 


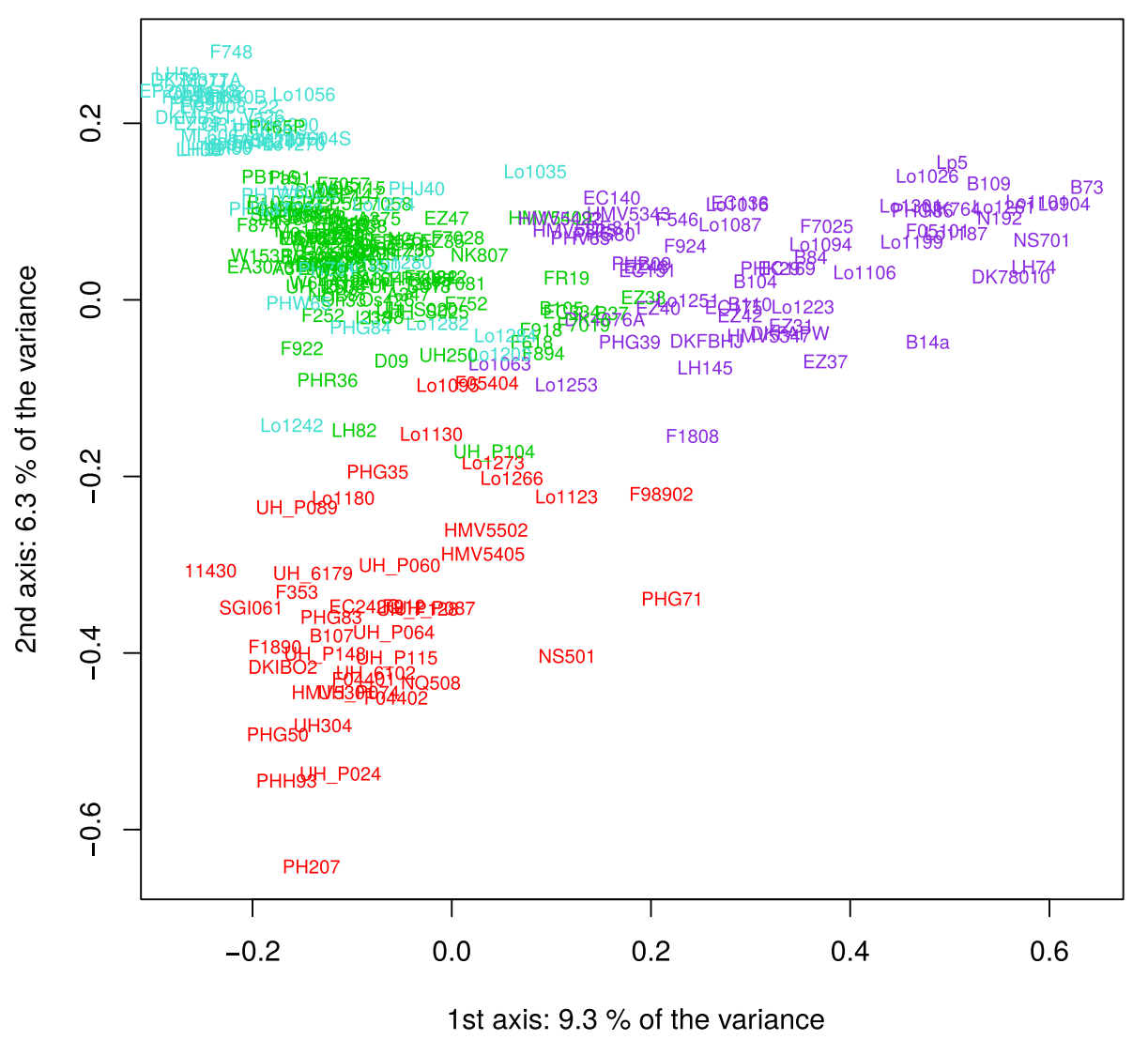

Fig. 2 Principal coordinate analysis (PCOA) of the DROPS panel. The PCOA was based on the covariance matrix K_Freq estimated from the $50 \mathrm{~K}$ Illumina array. The genetic groups identified by ADMIXTURE $\left(N_{Q}=4\right)$ are colored. Three key founders are found at extreme positions (lodent: PH207 in red, Stiff Stalk: B73 in violet, Lancaster: Mo17 in turquoise)

as illustrated in Fig. 1 on chromosome 3 (Table 2, and Additional file 3: Figure S3). For a LD extent of $r^{2} K=0.1,74,82$ and $89 \%$ of the physical map, and 42,58 and $71 \%$ of the genetic map were covered by the $50 \mathrm{~K}$, the GBS and the $600 \mathrm{~K}$, respectively (Table 2). For the combined data (ALL: $50 \mathrm{~K}+600 \mathrm{~K}+\mathrm{GBS})$, the coverage strongly varied between chromosomes, ranging from $83 \%$ (chromosome 7 ) to 98\% (chromosome 1) of the physical map, and from $51 \%$ (chromosome 7) to $97 \%$ (chromosome 1) of the genetic map (Table 2). For the physical map, increasing the LD extent threshold to $r^{2} K=0.4$ reduced the genome coverage from 89 to $49 \%$ for $600 \mathrm{~K}, 82$ to $28 \%$ for GBS, 74 to $20 \%$ for $50 \mathrm{~K}$ and 90 to $52 \%$ for the combined data. Increasing the MAF threshold reduced slightly the genome coverage, with smaller reduction for the physical map than genetic map. Surprisingly, increasing the SNP number by combining the markers from the arrays and GBS did not strongly increase the genome coverage as compared to the $600 \mathrm{~K}$, regardless of the threshold for LD extent (Fig. 1 and Additional file 3: Figure S3).

We observed a strong variation of genome coverage along each chromosome with contrasted patterns in low and high recombinogenic regions (Fig. 1 and Additional file 3: Figure S3). While low recombinogenic regions were totally covered with all the technologies (except for few intervals using the $50 \mathrm{~K}$ ), the genome coverage in high recombinogenic regions varied depending on both technology and SNP distribution. Forty-seven percent of the $2 \mathrm{Mbp}$ intervals in high recombination regions were better covered by the $600 \mathrm{~K}$ than the GBS against only $1 \%$, which were better covered by GBS than $600 \mathrm{~K}$. When exploring smaller window sizes $(20,100,500 \mathrm{~kb})$, the number of intervals better covered by $600 \mathrm{~K}$ than GBS decreased strongly when the intervals were shortened $(17.1 \%$ of $20 \mathrm{kbp}$-intervals vs $47.1 \%$ of $2 \mathrm{Mbp}$ intervals). In the contrary, the intervals better covered by GBS than $600 \mathrm{~K}$ increased slightly $(4.1 \%$ vs $1.1 \%$ of 2 Mbp-intervals). The number of interval with no or weak coverage differences between GBS and $600 \mathrm{~K}$ increased strongly: $84.5 \%$ of $20 \mathrm{kbp}$-intervals vs $68 \%$ of $2 \mathrm{Mbp}-$ intervals with coverage differences inferior to $10 \%$. Interestingly, the proportion of interval with strong coverage differences $(>50 \%)$ increased when the intervals were shortened $(7.8 \%$ of $20 \mathrm{kbp}$-intervals vs $0 \%$ of $2 \mathrm{Mbp}$ intervals). 


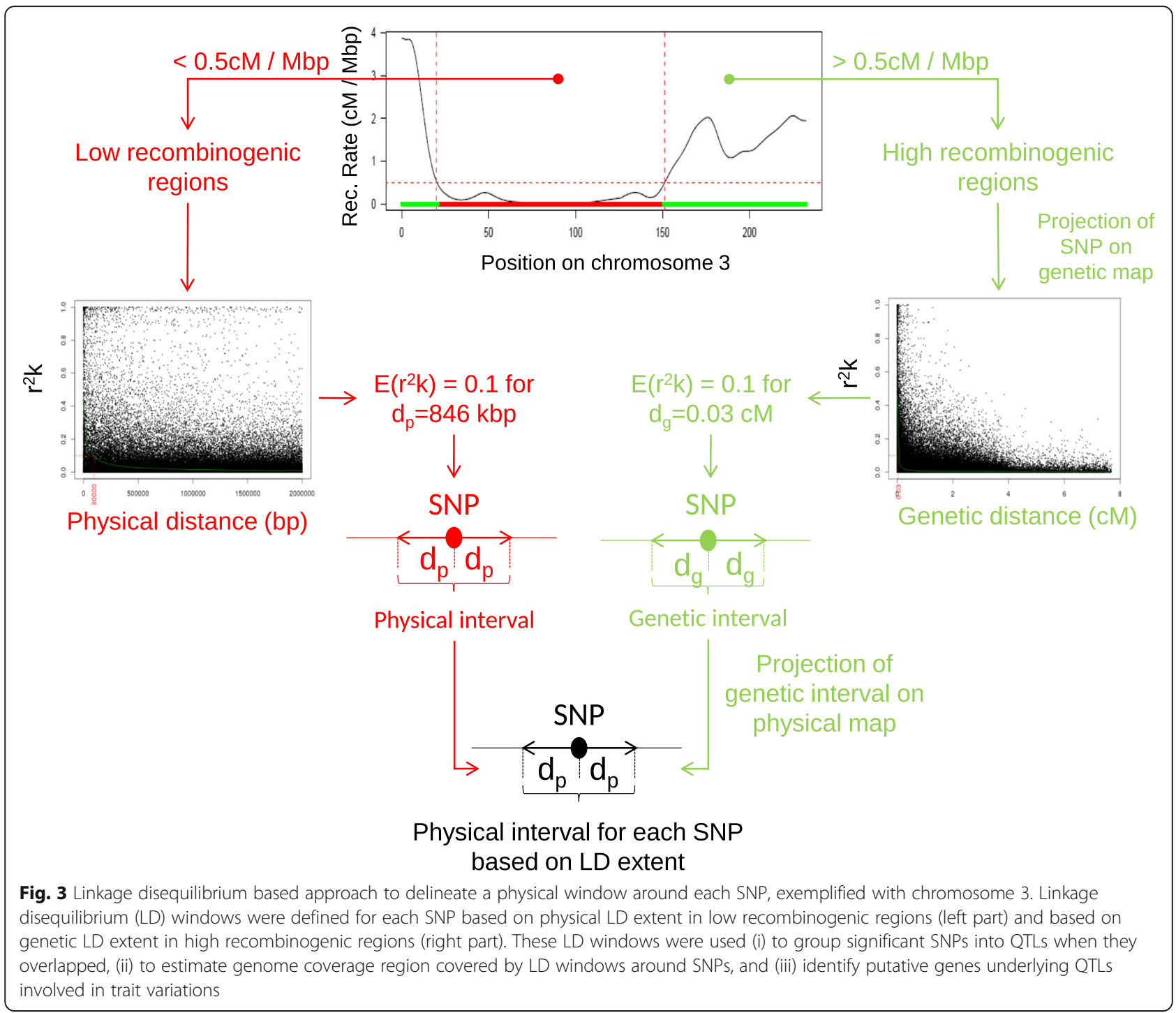

Number of QTLs detected using genome-wide association studies increases with markers density

We observed a strong variation in the number of SNPs significantly associated with the three traits across the 22 environments (Table 3). The mean number of significant SNPs per environment and trait was 3.7, 44.7, 17.9 and 62.4 for the $50 \mathrm{~K}, 600 \mathrm{~K}, \mathrm{GBS}$ and the three technologies combined, respectively (Table 4). Considering the $p$-value threshold used, 28, 303 and 204 false positives were expected among the $243,2,953$ and 1,182 associations detected for $50 \mathrm{~K}, 600 \mathrm{~K}$ and GBS, respectively. False discovery rate appeared therefore higher for GBS (17.2\%) than for DNA arrays (11.5 and $10.2 \%$ for $50 \mathrm{~K}$ and $600 \mathrm{~K}$, respectively). It can be explained by the higher genotyping error rate of GBS due to imputation and/or by its higher number of markers with a low MAF. Both reduce the power of GBS compared to DNA arrays and therefore lead to a higher false discovery rate. Proportionally to the SNP number, the $50 \mathrm{~K}$ and $600 \mathrm{~K}$ resulted in 1.5- and 1.7-fold more associated SNPs per situation (environment $\times$ trait) than GBS (p-value $<2 \times 10^{-6}$, Table 4). This difference between SNP-arrays and GBS was higher for grain yield (GY) and plant height (plantHT) than for male flowering time (DTA, Table 4).

We used two approaches based on LD for grouping significant SNPs (Fig. 3): (i) considering that all SNPs with overlapping LD windows for $r^{2} K=0.1$ belong to the same QTL (LD_win) and (ii) grouping significant SNPs that are adjacent on the physical map and are in LD $\left(r^{2} K>0.5, L D \_a d j\right)$. The QTLs defined by using the two approaches were globally consistent since significant SNPs within QTLs were in high LD whereas SNPs from different adjacent QTLs were not (Additional file 7: Figure S7-LDAdjacent and Additional file 8: Figure S8-LD-Windows). $L D \_$adj detected more QTLs than $L D \_$win for flowering 
Table 2 Variation of LD extent, and percentage of genome covered

\begin{tabular}{|c|c|c|c|c|c|c|c|c|c|c|c|c|}
\hline & & \multicolumn{10}{|c|}{ Chromosome } & \multirow{2}{*}{$\begin{array}{l}\text { Whole } \\
\text { Genome }\end{array}$} \\
\hline & & 1 & 2 & 3 & 4 & 5 & 6 & 7 & 8 & 9 & 10 & \\
\hline \multicolumn{2}{|l|}{ Physical Size (Mbp) } & 301 & 237 & 232 & 241 & 217 & 169 & 176 & 175 & 156 & 150 & 2,058 \\
\hline \multicolumn{2}{|l|}{ Genetic Size (cM) } & 268 & 211 & 188 & 150 & 205 & 129 & 148 & 182 & 145 & 139 & 1766 \\
\hline \multicolumn{2}{|c|}{ Physical LD extent (kbp) in low recombination regions } & 306 & 491 & 846 & 808 & 658 & 418 & 547 & 497 & 899 & 815 & 629 \\
\hline \multicolumn{2}{|c|}{ Genetic LD Extent (cM) in high recombination regions } & 0.062 & 0.027 & 0.033 & 0.022 & 0.031 & 0.019 & 0.012 & 0.038 & 0.023 & 0.019 & 0.029 \\
\hline \multirow[t]{4}{*}{ Percent of physical genome covered } & $50 \mathrm{~K}$ & $81 \%$ & $72 \%$ & $76 \%$ & $77 \%$ & $74 \%$ & $67 \%$ & $71 \%$ & $73 \%$ & $71 \%$ & $71 \%$ & $74 \%$ \\
\hline & $600 \mathrm{~K}$ & $98 \%$ & $88 \%$ & $91 \%$ & $89 \%$ & $90 \%$ & $84 \%$ & $81 \%$ & $90 \%$ & $87 \%$ & $84 \%$ & $89 \%$ \\
\hline & GBS & $92 \%$ & $81 \%$ & $84 \%$ & $83 \%$ & $83 \%$ & $77 \%$ & $77 \%$ & $81 \%$ & $79 \%$ & $76 \%$ & $82 \%$ \\
\hline & ALL & $98 \%$ & $90 \%$ & $92 \%$ & $90 \%$ & $91 \%$ & $87 \%$ & $83 \%$ & $92 \%$ & $88 \%$ & $85 \%$ & $90 \%$ \\
\hline \multirow[t]{4}{*}{ Percent of genetic map covered } & $50 \mathrm{~K}$ & $72 \%$ & $41 \%$ & $44 \%$ & $38 \%$ & $41 \%$ & $32 \%$ & $24 \%$ & $46 \%$ & $32 \%$ & $27 \%$ & $42 \%$ \\
\hline & $600 \mathrm{~K}$ & $96 \%$ & $71 \%$ & $76 \%$ & $68 \%$ & $72 \%$ & $62 \%$ & $47 \%$ & $78 \%$ & $63 \%$ & $53 \%$ & $71 \%$ \\
\hline & GBS & $86 \%$ & $58 \%$ & $61 \%$ & $53 \%$ & $61 \%$ & $48 \%$ & $37 \%$ & $63 \%$ & $48 \%$ & $40 \%$ & $58 \%$ \\
\hline & ALL & $97 \%$ & $74 \%$ & $78 \%$ & $72 \%$ & $74 \%$ & $65 \%$ & $51 \%$ & $81 \%$ & $66 \%$ & $57 \%$ & $74 \%$ \\
\hline
\end{tabular}

Genetic and Physical LD extent were obtained by adjusting Hill and Weir model's on 100 different sets of 500,000 loci randomly sampled in high (>0.5 cM / Mbp) and low $(<0.5 \mathrm{cM} / \mathrm{Mbp})$ recombination regions, respectively. The value represented the average across these 100 sets. The percentage of genome coverage was estimated using markers with MAF $>5 \%$ and $E\left(r^{2} K\right)=0.1$, for each technology and for the three technologies combined (ALL: GBS $+600 \mathrm{~K}+50 \mathrm{~K}$ )

time (242 vs 226), plant height (240 vs 160$)$ and grain yield (433 vs 237). The number of QTLs detected with the $L D_{-}$ adj approach increased strongly when the LD threshold was set above 0.5. Differences in QTL groupings between the two methods were observed for specific LD and recombination patterns. This occurred for instance on chromosome 6 for grain yield (Additional file 7: Figure S7-LD-Adjacent and Additional file 8: Figure S8-LD-Windows). Within this region, the recombination rate was low and the LD pattern between associated SNPs was complex (Additional file 3: Figure S3). While $L D \_$adj splitted several SNPs in high LD into different QTLs (for instance QTL 232, 235, 237, 249), $L D \_$win grouped together associated SNPs that are genetically close but displayed a low LD (Additional file 7: Figure S7-LD-Adjacent and Additional file 8: Figure S8-LDWindows). Reciprocally, for flowering time, we observed different cases where $L D_{-}$win separated distant SNPs in high LD into different QTLs, whereas $L D \_a d j$ grouped them (QTL 25 and 26, 51 to 53, 95 to 97, 208 and 209, 218 and 219). As these differences were specific to complex LD and recombination patterns, we used the $L D_{-}$win approach for the rest of the analyses.

Although a large difference in number of associated SNPs was observed between $600 \mathrm{~K}$ and GBS, little difference was observed between QTL number after grouping SNPs (Table 3, Table 4). The mean number of QTLs was indeed 1.0, 5.9, 5.2 and 9.5 for the $50 \mathrm{~K}, 600 \mathrm{~K}$, GBS, and the three technologies combined, respectively (Table 4 ). Note that the number of QTLs continued to increase with marker density when SNPs from GBS, $50 \mathrm{~K}$ and $600 \mathrm{~K}$ were combined (Additional file 9: Figure S9). The number of SNPs associated with each QTL varied according to the technology (on average 3.7, 7.6, 3.4 and
6.6 significant SNPs for the $50 \mathrm{~K}, 600 \mathrm{~K}$, GBS, and the combined technologies, respectively). The total number of QTLs detected over all environments using the $600 \mathrm{~K}$ and GBS was close for flowering time (130 vs 133) and plant height (96 vs 90). It was 1.4-fold higher for the $600 \mathrm{~K}$ than GBS for grain yield (166 vs 120).

\section{The $600 \mathrm{~K}$ and GBS were highly complementary for association mapping}

Seventy-eight percent, 76 and 71\% of the QTLs of flowering time, plant height and grain yield were specifically detected by the $600 \mathrm{~K}$ or GBS, respectively (Fig. 4). On the contrary, the $50 \mathrm{~K}$ displayed very few specific QTLs. When we combined the GBS and $600 \mathrm{~K}$ markers, $7 \%$ of their common QTLs had $-\log _{10}($ Pval) increased by 2 and $21 \%$ by 1 , potentially indicating a gain in accuracy of the position of the causal polymorphism (Additional file 10: Table S1).

This complementarity between GBS and $600 \mathrm{~K}$ is well exemplified with two strong association peaks for flowering time on chromosome 1 (QTL32) and 3 (QTL95) detected in several environments (Additional file 10: Table S1 and Fig. 5a). In order to better understand the origin of the complementarity between GBS and $600 \mathrm{~K}$ technologies for GWAS, we scrutinized the LD between SNPs and the haplotypes within these two QTLs (Fig. 5b and c, and Additional file 11: Figure S10 for other examples). QTL95 showed a gain in power. It was only identified by the $600 \mathrm{~K}$ although the region included numerous SNPs from GBS close to the associated peak. None of these SNPs were in high LD with the most associated marker of the QTL95 (Fig. 5b). QTL32 was detected by 1 to 10 GBS markers in 9 environments with 
Table 3 Number of significant SNPs per environment, per technology and for the combined technologies

\begin{tabular}{|c|c|c|c|c|c|c|c|c|c|c|c|c|c|c|c|}
\hline \multirow[b]{2}{*}{ Env. } & \multicolumn{5}{|c|}{ Flowering Time } & \multicolumn{5}{|c|}{ Plant Height } & \multicolumn{5}{|c|}{ Grain Yield } \\
\hline & Herit. & $50 \mathrm{~K}$ & $600 \mathrm{~K}$ & GBS & $\mathrm{ALL}$ & Herit. & $50 \mathrm{~K}$ & $600 \mathrm{~K}$ & GBS & ALL & Herit. & $50 \mathrm{~K}$ & $600 \mathrm{~K}$ & GBS & ALL \\
\hline Cam12R & 0.40 & 0 & 3 & 1 & 4 & 0.57 & 23 & 209 & 88 & 289 & 0.37 & 21 & 286 & 102 & 381 \\
\hline Cam12W & 0.60 & 1 & 18 & 13 & 31 & 0.39 & 22 & 270 & 72 & 339 & 0.54 & 41 & 525 & 167 & 684 \\
\hline Cam13R & 0.46 & 0 & 9 & 7 & 16 & 0.21 & 0 & 2 & 3 & 5 & 0.19 & 0 & 1 & 3 & 4 \\
\hline Cra12R & 0.69 & 1 & 40 & 18 & 59 & 0.32 & 0 & 6 & 3 & 9 & 0.25 & 3 & 57 & 45 & 103 \\
\hline Cra12W & 0.72 & 3 & 25 & 19 & 43 & 0.19 & 10 & 69 & 16 & 83 & 0.53 & 12 & 98 & 53 & 150 \\
\hline Deb12R & 0.63 & 1 & 14 & 16 & 30 & 0.33 & 0 & 1 & 0 & 1 & 0.57 & 2 & 14 & 0 & 16 \\
\hline Deb12W & 0.71 & 0 & 25 & 38 & 61 & 0.37 & 0 & 6 & 7 & 7 & 0.47 & 0 & 6 & 2 & 8 \\
\hline Deb13R & 0.59 & 1 & 17 & 5 & 23 & 0.08 & 0 & 33 & 9 & 42 & 0.37 & 1 & 22 & 15 & 37 \\
\hline Gai12R & 0.64 & 8 & 80 & 24 & 104 & 0.18 & 1 & 47 & 41 & 89 & 0.31 & 0 & 23 & 8 & 31 \\
\hline Gai12W & 0.66 & 5 & 42 & 15 & 59 & 0.46 & 0 & 1 & 3 & 4 & 0.58 & 3 & 71 & 14 & 85 \\
\hline Gai13R & 0.62 & 0 & 24 & 8 & 31 & 0.66 & 0 & 6 & 6 & 11 & 0.63 & 0 & 4 & 5 & 9 \\
\hline Gai13W & 0.73 & 1 & 45 & 9 & 54 & 0.33 & 0 & 1 & 3 & 4 & 0.76 & 2 & 7 & 1 & 9 \\
\hline Kar12R & 0.72 & 4 & 30 & 21 & 52 & 0.30 & 0 & 4 & 3 & 7 & 0.71 & 0 & 5 & 6 & 11 \\
\hline Kar12W & 0.77 & 8 & 60 & 10 & 73 & 0.23 & 1 & 10 & 4 & 14 & 0.53 & 2 & 19 & 11 & 29 \\
\hline Kar13R & 0.68 & 3 & 65 & 11 & 77 & 0.31 & 0 & 4 & 2 & 6 & 0.75 & 4 & 37 & 24 & 62 \\
\hline Kar13W & 0.73 & 0 & 17 & 12 & 29 & 0.26 & 0 & 2 & 7 & 9 & 0.67 & 4 & 12 & 6 & 19 \\
\hline Mur13R & 0.83 & 3 & 48 & 19 & 68 & 0.32 & 7 & 61 & 7 & 68 & 0.84 & 14 & 90 & 28 & 116 \\
\hline Mur13W & 0.76 & 0 & 11 & 8 & 19 & 0.32 & 3 & 4 & 2 & 9 & 0.74 & 10 & 80 & 25 & 104 \\
\hline Ner12R & 0.72 & 7 & 23 & 18 & 45 & 0.34 & 0 & 7 & 3 & 10 & 0.54 & 1 & 10 & 6 & 16 \\
\hline Ner12W & 0.81 & 1 & 80 & 30 & 107 & 0.24 & 0 & 2 & 2 & 4 & 0.59 & 1 & 8 & 6 & 15 \\
\hline Ner13R & 0.76 & 3 & 60 & 26 & 88 & 0.26 & 1 & 25 & 13 & 38 & 0.32 & 0 & 13 & 7 & 20 \\
\hline Ner13W & 0.81 & 2 & 23 & 17 & 42 & 0.20 & 0 & 8 & 5 & 13 & 0.73 & 2 & 28 & 4 & 32 \\
\hline Average & 0.68 & 2.4 & 34.5 & 15.7 & 50.7 & 0.31 & 3.1 & 35.4 & 13.6 & 48.2 & 0.55 & 5.6 & 64.4 & 24.5 & 88.2 \\
\hline Median & 0.71 & 1 & 25 & 15.5 & 47.5 & 0.32 & 0 & 6 & 4.5 & 9.5 & 0.56 & 2 & 20.5 & 7.5 & 30 \\
\hline SD & 0.11 & 2.6 & 22.7 & 8.7 & 27.7 & 0.13 & 6.8 & 69.6 & 23.2 & 90.2 & 0.18 & 9.6 & 120.1 & 39.5 & 156.8 \\
\hline
\end{tabular}

The average, median and standard deviation (SD) per environment are calculated for each trait (male Flowering Time, Plant Height, Grain Yield). "Herit.": narrow sense heritability. "Env.": Environment

Table 4 Comparison of associated SNPs and QTLs detected between traits and three technologies

\begin{tabular}{|c|c|c|c|c|c|c|c|c|c|}
\hline & & \multicolumn{4}{|c|}{ Significant SNPs } & \multicolumn{4}{|l|}{ QTLs } \\
\hline \multicolumn{2}{|l|}{ Technology } & $50 \mathrm{~K}$ & $600 \mathrm{~K}$ & GBS & ALL & $50 \mathrm{~K}$ & $600 \mathrm{~K}$ & GBS & ALL \\
\hline \multicolumn{2}{|l|}{ Marker Nb } & 42046 & 459191 & 308929 & 810580 & 42046 & 459191 & 308929 & 810580 \\
\hline \multirow[t]{4}{*}{ Total Nb } & DTA & 52 & 759 & 345 & 1115 & 20 & 130 & 133 & 226 \\
\hline & plantHT & 68 & 778 & 299 & 1061 & 16 & 96 & 90 & 160 \\
\hline & GY & 123 & 1416 & 538 & 1941 & 33 & 166 & 120 & 238 \\
\hline & Per trait & 81 & 984 & 394 & 1372 & 23 & 131 & 114 & 208 \\
\hline \multirow[t]{4}{*}{ Average per envir. } & DTA & 2.4 & 34.5 & 15.7 & 50.7 & 0.9 & 5.9 & 6.0 & 10.3 \\
\hline & plantHT & 3.1 & 35.4 & 13.6 & 48.2 & 0.7 & 4.4 & 4.1 & 7.3 \\
\hline & GY & 5.6 & 64.4 & 24.5 & 88.2 & 1.5 & 7.5 & 5.5 & 10.8 \\
\hline & Per trait & 3.7 & 44.7 & 17.9 & 62.4 & 1.0 & 5.9 & 5.2 & 9.5 \\
\hline
\end{tabular}

QTLs were obtained by grouping associated SNPs with overlapping LD windows (LD_win) for the three traits (DTA male flowering time, PlantHT plant height, GY grain yield). "Marker Nb" indicates the number of markers tested in GWAS. "Total number": is the sum of associated SNPs or QTLs across environments. "Average per envir" indicates the average number of QTLs obtained in 22 environments for three traits (66 trait-environments combinations) 
Male Flowering Time

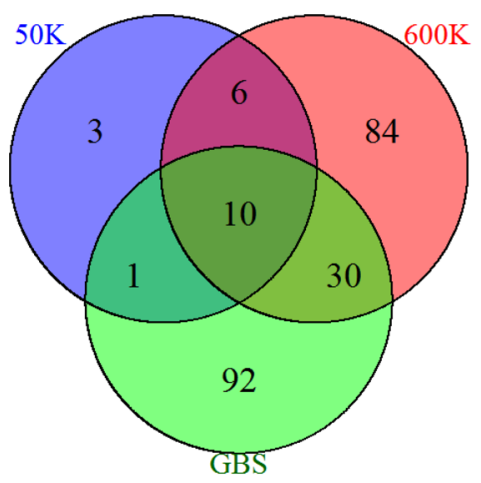

Plant Height

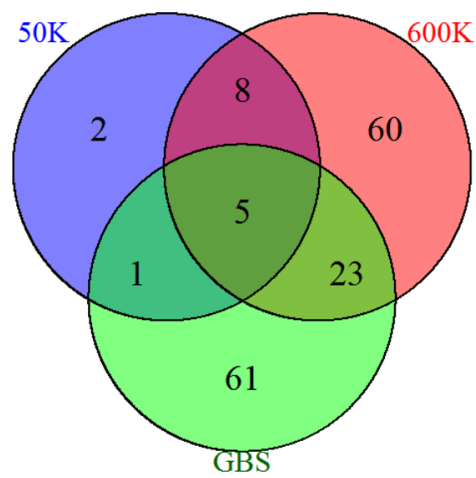

Grain Yield

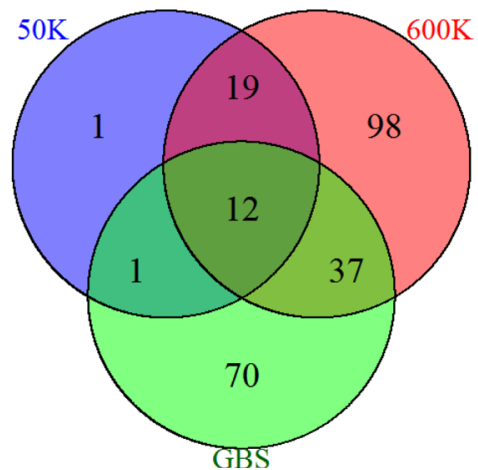

Fig. 4 Complementarity of the three technologies to detect QTLs. The numbers of specific QTLs detected by each technology for the three traits (flowering time, plant height, grain yield) are shown

$-\log (p$-value $)$ ranging from 5 to 7.6 , whereas it was detected by only two $600 \mathrm{~K}$ markers in one environment (Ner12W) with $-\log (p$-value) slightly above the significance threshold (Additional file 10: Table S1 and Fig. 5b).

Haplotype analyses showed that the SNPs from the GBS within QTL95 were not able to discriminate all haplotypes (Fig. 5c). In QTL95, the $600 \mathrm{~K}$ markers discriminated the three main haplotypes $(\mathrm{H} 1, \mathrm{H} 2, \mathrm{H} 3)$, whereas the GBS markers did not discriminate H3 against $\mathrm{H} 1+\mathrm{H} 2$. As $\mathrm{H} 1$ contributed to an earlier flowering time than $\mathrm{H} 2$ or $\mathrm{H} 3$, associations appeared more significant for the $600 \mathrm{~K}$ than for GBS (Fig. 5c). In QTL32, the use of GBS markers identified late individuals that mostly displayed $\mathrm{H} 1, \mathrm{H} 2$ and $\mathrm{H} 3$ haplotypes, against early individuals that mostly displayed $\mathrm{H} 4$ and $\mathrm{H} 5$ haplotypes (Fig. 5c). The gain of power for GBS markers as compared to $600 \mathrm{~K}$ markers for QTL32 originated from the ability to discriminate late individuals (black alleles) from early individuals (red alleles) within $\mathrm{H} 4$ haplotypes (Fig. 5c).

To further decipher the GWAS differences between $600 \mathrm{~K}$ and GBS, we used a resampling approach to explore the interplay between (i) MAF distribution and (ii) SNP distribution along the genome, at different SNP densities. We detected more SNP associations, but less QTLs with a MAF distribution skewed towards low than high MAF. This difference increased as marker density increased (Additional file 12: Figure S11). As GBS has a MAF distribution skewed towards low MAF compared to $600 \mathrm{~K}$, GBS detected more QTLs but less associated SNPs than $600 \mathrm{~K}$. This discrepancy between association and QTL detection came from the fact that QTLs with low MAF were identified by less associated SNP than those with high MAF (Additional file 13: Figure S12).

SNPs distributed similarly to GBS detected more QTLs but less significant SNPs than those following the distribution of the $600 \mathrm{~K}$ and $50 \mathrm{~K}$, notably for the highest SNP density (Additional file 13: Figure S12). We observed that SNPs evenly distributed according to the physical distance detected more associations, but less QTLs than all other SNP distributions along the genome. It was the contrary for SNPs evenly distributed according to genetic distance (Additional file 12: Figure S11 C and D). This is consistent with QTL distribution along the genome being more correlated to the genetic than physical distance (see below), and the fact that recombination is higher in gene rich regions, leading to less associated SNPs per QTL. Superiority of QTL detection by the GBS distribution as compared 


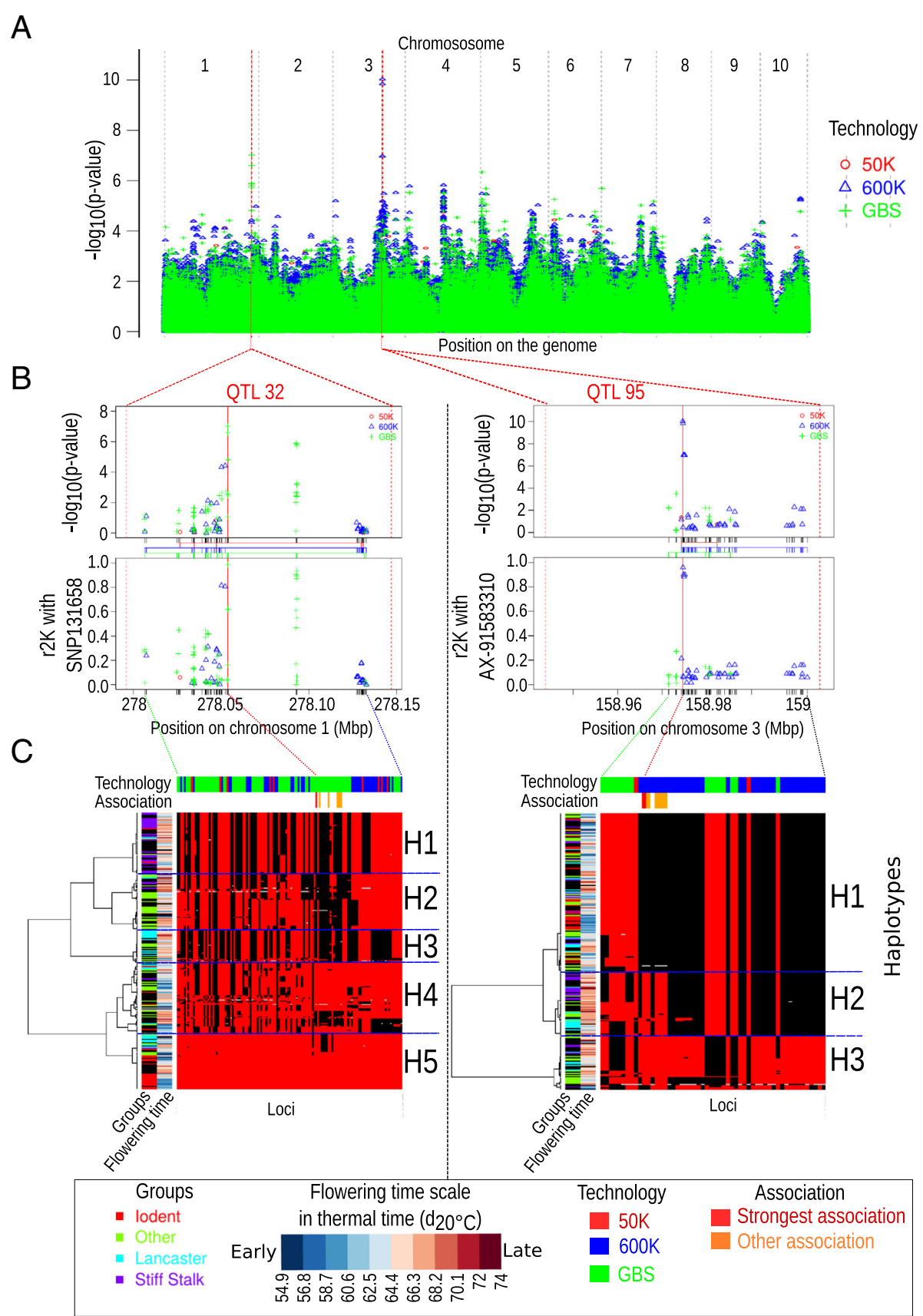

Fig. 5 Complementarity of QTLs detection between the $600 \mathrm{~K}$ array and the GBS for two regions (QTL 32/QTL95). a Manhattan plot of the $-\log _{10}(p$-value) along the genome. Dotted red lines correspond to QTL32 and QTL95 located on chromosome 1 and 3, respectively, for the flowering time in one environment (Ner13R). b Local manhattan plot of the - $\log _{10}(p$-value) (top) and linkage disequilibrium corrected by the kinship $\left(r^{2} K\right)$ (bottom) of all SNPs with the strongest associated marker within QTL 32 (left) and QTL 95 (right). Colored vertical lines between manhattan plot and linkage disequilibrium plot represents the distribution of markers for different technologies. Dotted lines between panels $b$ and c linked the first marker, the most associated marker, and the last marker of each QTL (c) Local haplotypes displayed by all SNPS within the QTLS 32 (left) and 95 (right) with MAF > 5\%. Inbred lines are in rows and SNPs are in columns. Inbred lines were ordered by hierarchical clustering based on local dissimilarity estimated by all SNPs within each QTL. Genotyping matrix is colored according to their allelic dose at each SNP. Red and black represent homozygotes and gray represent heterozygotes. The associated peaks (red vertical lines) and other associated SNPs with $-\log _{10}(p$-value $)>5$ (orange vertical lines) are indicated above the genotyping matrix. H1, H2, H3, H4, H5 represent the 5 and 3 haplotypes obtained by cutting the dendograms with the most 5 and 3 dissimilar clusters within QTL32 and QTL95, respectively 
to the $600 \mathrm{~K}$ and $50 \mathrm{~K}$ SNP distributions came from the higher proportion of SNPs in high recombinogenic regions for GBS than for $600 \mathrm{~K}$ and $50 \mathrm{~K}$ (Fig. 1). This suggests that the complementarity of $600 \mathrm{~K}$ and GBS in terms of QTL detected and SNP associations came also from their specificities for both SNP distributions along the genome and MAF distribution. In the end, we studied the impact of genomic coverage differences between $600 \mathrm{~K}$ and GBS on QTL detection along the genome. QTLs detected by both $600 \mathrm{~K}$ and GBS were located in intervals with large differences in coverage less frequently than their proportion in the entire genome $(0.8 \%$ vs $7.8 \%$, respectively). Intervals with specific QTLs showed an enrichment in such intervals with high differences in coverage (3.5\%), but still below the proportion in the entire genome. It confirms that most specific QTLs showed no strong genomic coverage differences between GBS and $600 \mathrm{~K}$ and therefore that complementarity of QTL detection between these two technologies came from the ability to tag different haplotypes.

\section{Colocalization of QTLs between environments and traits and distribution of QTLs along the genome}

After combining the three technologies, we identified 226, 160, 238 QTLs for flowering time, plant height and grain yield, respectively (Table 4 and Additional file 10: Table S1). We highlighted 23 QTLs with the strongest effects on flowering time, plant height and grain yield $\left(-\log _{10}(\right.$ Pval $) \geq 8$, Table 5$)$. The strongest association corresponded to the QTL95 for flowering time $\left(-\log _{10}(p-\right.$ value $)=10.03)$ on chromosome $3(158,943,646-159$, $005,990 \mathrm{bp})$, the QTL135 for GY $\left(-\log _{10}(p\right.$-value $\left.)=18.7\right)$ on chromosome $6(12,258,527-29,438,316 \mathrm{bp})$ and QTL78 on chromosome $6(12,258,527-20,758,095$ bp $)$ for plant height $\left(-\log _{10}(p\right.$-value $\left.)=17.31\right)$. The QTL95 for flowering time trait was the most stable QTLs across

Table 5 Summary of the main QTLs $\left(-\log _{10}(\right.$ Pval $\left.) \geq 8\right)$ identified for the three traits

\begin{tabular}{|c|c|c|c|c|c|c|c|c|c|c|c|c|c|}
\hline Trait & QTL & Chr & Pos & Lower Limit & Upper Limit & R2 & Effect & Log & Minor All & Major All & MAF & EnvMax & $\mathrm{Nb}$ DiffEnv \\
\hline$\overline{\text { DTA }}$ & 95 & 3 & $158,974,594$ & $158,943,646$ & $159,005,990$ & 0.15 & 1.27 & 10.03 & $G$ & $C$ & 0.41 & Ner13R & 19 \\
\hline \multirow[t]{9}{*}{ plantHT } & 21 & 2 & $130,441,738$ & $129,971,437$ & $130,912,039$ & 0.15 & -6.74 & 9.21 & A & G & 0.14 & Cam12W & 3 \\
\hline & 71 & 6 & $6,614,012$ & $6,593,785$ & $6,636,807$ & 0.13 & -4.76 & 8.39 & G & A & 0.18 & Cam12R & 2 \\
\hline & 72 & 6 & $6,807,230$ & $6,793,841$ & $6,837,747$ & 0.14 & -4.87 & 8.77 & T & G & 0.18 & Cam12R & 2 \\
\hline & 78 & 6 & $20,330,595$ & $12,258,527$ & $20,758,095$ & 0.27 & -8.99 & 17.31 & C & T & 0.26 & Cam12W & 4 \\
\hline & 79 & 6 & $22,905,376$ & $21,037,721$ & $23,951,687$ & 0.19 & -5.45 & 11.42 & T & G & 0.31 & Cam12R & 3 \\
\hline & 80 & 6 & $25,3178,25$ & $24,184,017$ & $26,606,537$ & 0.13 & -4.41 & 8.17 & T & C & 0.2 & Cam12R & 2 \\
\hline & 81 & 6 & $28,130,108$ & $26,695,327$ & $28,659,766$ & 0.16 & -5.14 & 9.84 & C & G & 0.44 & Cam12R & 2 \\
\hline & 94 & 6 & $101,482,646$ & $101,463,249$ & $101,501,936$ & 0.14 & -5.98 & 8.22 & T & A & 0.17 & Cam12W & 2 \\
\hline & 110 & 8 & $12,782,777$ & $12,767,198$ & $12,798,330$ & 0.19 & -7.67 & 12.44 & C & T & 0.22 & Cam12W & 3 \\
\hline \multirow[t]{13}{*}{ GY } & 65 & 3 & $141,621,777$ & $140,505,559$ & $144,210,207$ & 0.12 & 0.42 & 8.13 & A & C & 0.27 & Gai12W & 5 \\
\hline & 85 & 3 & $187,028,970$ & $186,994,852$ & 187057,772 & 0.12 & -0.48 & 8.49 & A & C & 0.28 & Kar12W & 1 \\
\hline & 120 & 6 & $5,155,708$ & $5,131,927$ & $5,177,694$ & 0.12 & -0.58 & 8.24 & C & $\mathrm{T}$ & 0.42 & Cam12W & 2 \\
\hline & 122 & 6 & $5,638,516$ & $5,623,945$ & $5,659,803$ & 0.12 & -0.57 & 8.11 & $\mathrm{~T}$ & G & 0.23 & Cam12W & 2 \\
\hline & 124 & 6 & $5,871,000$ & $5,855,407$ & $5,887,383$ & 0.12 & -0.56 & 8.4 & T & G & 0.42 & Cam12W & 2 \\
\hline & 127 & 6 & $6,612,654$ & $6,593,785$ & $6,636,807$ & 0.16 & -0.65 & 10.44 & C & A & 0.32 & Cam12W & 2 \\
\hline & 128 & 6 & $6,807,462$ & $6,793,841$ & $6,837,747$ & 0.12 & -0.54 & 8.41 & A & C & 0.26 & Cam12W & 2 \\
\hline & 129 & 6 & $6,890,199$ & $6,878,877$ & $6,930,838$ & 0.12 & 0.63 & 8.06 & T & A & 0.48 & Cam12W & 3 \\
\hline & 130 & 6 & $7,046,773$ & $7,027,497$ & $7,088,575$ & 0.13 & 0.62 & 8.71 & A & G & 0.4 & Cam12W & 3 \\
\hline & 131 & 6 & $7,159,714$ & $7,113,662$ & $7,200,479$ & 0.12 & 0.59 & 8.24 & T & C & 0.39 & Cam12W & 3 \\
\hline & 135 & 6 & $18,528,943$ & $12,258,527$ & $29,438,316$ & 0.28 & -0.78 & 18.7 & G & C & 0.31 & Cam12W & 6 \\
\hline & 147 & 6 & $101,482,646$ & $101,463,249$ & $101,501,936$ & 0.22 & -0.65 & 15.04 & T & A & 0.17 & Cam12W & 5 \\
\hline & 173 & 8 & $12,782,777$ & $12,767,198$ & $12,798,330$ & 0.17 & -0.61 & 11.8 & C & $\mathrm{T}$ & 0.22 & Cam12W & 4 \\
\hline
\end{tabular}

"Pos" indicates the physical position in base pair of the SNP with the strongest association on the V2 of reference genome. LowerLimit and UpperLimit indicate the lower and upper physical limits estimated by LD windows (LD_win) for each QTL. The proportion of the variance explained ( $\mathrm{R}^{2}$ ), the effect of the major allele (Effect) as outputted by FastLMM, $-\log _{10}$ (Pval) (Log), the minor and major alleles (Minor All and Major All) and the minor allele frequency (MAF) of the most significant SNP within the QTL are shown. The following columns represent environment for which the most associated QTL was observed (EnvMax) and the number of different environments in which QTL are detected (NbDiffEnv) are shown. Note that QTLs 71-72 for the plant height and QTLs 129-130 for the grain yield are genetically close $(<1 \mathrm{cM})$ and display high mean LD $\left(r^{2} K>0.5\right)$. Hence, QTLs $71-72$ and $129-130$ can potentially be merged 
environments since it was detected in 19 environments (Additional file 10: Table S1). Moreover, this QTL showed a colocalization with QTL74 for grain yield in 5 environments and QTL30 for plant height in 1 environment suggesting a pleiotropic effect. More globally, 472 QTLs appeared trait-specific whereas 70 QTLs overlapped between at least two traits $(6,3,5.2$ and $3.0 \%$ for GY and plantHT, GY and DTA, and DTA and plantHT, respectively) suggesting that some QTLs may be pleiotropic (Additional file 14: Figure S13). This was not surprising since average corresponding correlations within environments for these traits were moderate $(0.47,0.54$ and 0.45 , respectively). Only $0.7 \%$ overlapped between the three traits (Additional file 14: Figure S13). Twenty percent of QTLs were detected in at least two environments and $9 \%$ in at least three environments (Additional file 15: Table S2). We observed no significant differences of stability between the three traits $(p$-value $=0.2)$. However, 6 out 7 most stable QTLs (Number of environments $>5$ ) were found for flowering time. This was consistent with higher average correlations between environments observed for flowering time than for plant height and grain yield (0.76, $0.43,0.48$, respectively). We observed that QTLs that displayed a significant effect in more than one environment had larger effects and $-\log (p$-value $)$ values than those significant in a single environment. This difference in -log(p-value) values was stronger for grain yield and plant height than flowering time.

The distribution of QTLs was not homogeneous along the genome since 82,77 and $79 \%$ of flowering time, plant height and grain yield QTLs, respectively, were located in the high recombinogenic regions, whereas they represented $46 \%$ of the physical genome (Additional file 16: Table S3). The QTLs were more stable ( $\geq 2$ environments) in low than in high recombinogenic regions $(12.8 \%$ vs $5.8 \%$, $p$-value $=0.03)$.

\section{Discussion \\ GBS required massive imputation but displayed similar global trends than DNA arrays for genetic diversity organization}

In order to reduce genotyping cost, GBS is most often performed at low depth leading to a high proportion of missing data, thereby requiring imputation in order to perform GWAS. Imputation can produce genotyping errors that can cause false associations and introduce bias in diversity analysis [33]. We evaluated the quality of genotyping and imputation obtained by different approaches, taking the $50 \mathrm{~K}$ or $600 \mathrm{~K}$ as references. The best imputation method that yielded a fully genotyped matrix with the lowest error rate for the prediction of both heterozygotes and homozygotes was the approach merging the homozygous genotypes from TASSEL and the imputation of Beagle for the other data $\left(\mathrm{GBS}_{5}\right.$ Table 1$)$.
The quality of imputation was high with $96 \%$ of allelic values consistent with those of the $50 \mathrm{~K}$ and $600 \mathrm{~K}$. This level of concordance is identical to a study of USA national maize inbred seed bank by Romay et al. [32]. It is higher than in a diversity study of European flint maize collection (93\%) by Gouesnard et al. [33], which was more distant from the reference AllZeaGBSv2.7 database than for the panel presented here. For further studies, integrating genotyping data from the three technologies may reduce imputation errors for missing data of GBS [35].

The ascertainment bias of SNP-arrays due to the limited number of lines used for SNP discovery was reinforced by counter-selection of rare alleles during the design process of DNA arrays [3, 4]. For GBS, the polymorphism database to call polymorphisms included thousands of diverse lines [38]. In our study, we used AllZeaGBSv2.7 database. After a first step of GBS imputation $\left(\mathrm{GBS}_{2}\right)$, missing data dropped to $11.9 \%$ i.e. only slightly more than in Romay et al. (10\%) [32]. This confirms that the polymorphism database (AllZeaGBSv2.7) covered adequately the genetic diversity of our genetic material.

Although, we observed differences of allelic frequency spectrum between GBS and DNA arrays, these technologies revealed similar trends in the organization of population structure and relatedness (Fig. 2, Additional file 4: Figure S4) suggesting no strong ascertainment bias for deciphering global genetic structure trends in the panel. However, although highly correlated, level of relatedness differed between GBS and DNA arrays, especially when the lines were less related as showed by the deviation (to the left) of the linear regression from the bisector (Additional file 5: Figure S5).

\section{The extent of linkage disequilibrium strongly varied along and between chromosomes}

Linkage disequilibrium extent in high recombinogenic regions varied to a large extent among chromosomes, ranging from 0.012 to $0.062 \mathrm{cM}$. Similar variation of genetic LD extent between maize chromosomes has been previously observed by Rincent et al. [14], but their classification of chromosomes was different from ours. This difference could be explained by the fact that we analyzed specifically high and low recombination regions. According to Hill and Weir Model [39], the physical LD extent in a genomic region increased when the local recombination rate decreased. As a consequence, chromosome 1 and 9 had the lowest and highest physical LD extent and displayed the highest and one of the lowest recombination rate in pericentromeric regions, respectively $(0.26$ vs $0.11 \mathrm{cM} / \mathrm{Mbp}$, Table 2 and Additional file 16: Table S3). Unexpectedly, the genetic LD extent also correlated negatively with the 
recombination rate. It suggested that chromosomes with a low recombination rate also display a low effective population size. Background selection against deleterious alleles could explain this pattern since it reduces the genetic diversity in low recombinogenic regions $[40,41]$. Finally, we observed a strong variation of the LD extent along each chromosome. As we used a consensus genetic map [42] that represents well the recombination within our population, it suggested, according to Hill and Weir's model, that the number of ancestors contributing to genetic diversity varied strongly along the chromosomes. This likely reflects the selection of genomic regions for adaptation to environment or agronomic traits [40], that leads to a differential contribution of ancestors according to their allelic effects. Ancestors with strong favorable allele(s) in a genomic region may lead ultimately to large identical by descent genomic segments [43].

\section{SNPs were clustered into QTL highlighting interesting genomic regions}

In previous GWAS studies, the closest associated SNPs were grouped into QTLs according to either a fixed physical distance [1] or a fixed genetic distance [29, 44]. These approaches suffer of two drawbacks. First, the physical LD extent can vary strongly along chromosomes according to the variation of recombination rate (Fig. 1 and Additional file 3: Figure S3). Second, the genetic LD extent depends both on panel composition and the position along the genome (Table 2). These approaches may therefore strongly overestimate or underestimate the number of QTLs. To address both issues Cormier et al. [45] proposed to group associated SNPs by using a genetic window based on the genetic LD extent estimated by Hill and Weir model in the genomic regions around the associated peaks [39]. In our study, we improved this last approach (LD_win):

- First, we used $r^{2} K$ that corrected $r^{2}$ for kinship rather than the classical $\mathrm{r}^{2}$ since $r^{2} K$ reflected the LD addressed in our GWAS mixed models to map QTL [17].

- Second, we took advantage of the availability of both physical and genetic maps of maize to project the genetic LD extent on the physical map. This physical window was useful to retrieve the annotation from B73 reference genome, decipher local haplotype diversity (Fig. 5) and estimate physical genome coverage (Table 2, Additional file 3: Figure S3).

- Third, we considered an average LD extent estimated separately in the high and low recombinogenic genomic regions. This average was estimated by using several large random sets of pairs of loci in these regions rather than the local LD extent in the genomic regions around each associated peaks.

We preferred this approach rather than using local LD extent in order to limit the effect of (i) the strong variation of marker density along the chromosome (Additional file 3: Figure S3), (ii) the local ascertainment bias due to the markers sampling (iii) the poor estimation of the local recombination rate using a genetic map, notably for low recombination regions [3, 43], and (iv) errors in locus order due to assembly errors or chromosomal rearrangements.

We compared $L D \_$win with $L D_{-}$adj, another approach based on LD to group the SNPs associated to trait variation into QTLs. The discrepancies between the two approaches can be explained by the local recombination rate and LD pattern. Since $L D_{-}$adj approach was based on the grouping of contiguous SNPs according to their LD, this approach was highly sensitive to (i) errors in marker order or position due to genome assembly errors or structural variations, which are important in maize [46] (ii) genotyping or imputation errors, which we estimated at ca. $1 \%$ and ca. 4\%, respectively, for GBS (Table 1), (iii) presence of allelic series with contrasted effects in different experiments which are currently observed in maize [42], (iv) LD threshold used. On the other hand, $L D_{-}$win lead either to inflate the number of QTLs in high recombinogenic regions in which SNPs were too distant genetically to be grouped, or deflated their number by grouping associated SNPs in low recombinogenic regions. Since $L D_{-} w i n$ considered the average LD extent, this method could conduct either to separate or group abusively SNPs when local LD extent was different than the global LD extent. Simulations will be carried out in further research to better understand the properties of $L D_{-}$win and $L D_{-}$adj approaches.

Note that LD windows should not be considered as confidence intervals since the relationship between LD and recombination is complex due to demography, drift and selection in association panels, contrary to linkage based QTL mapping [17]. The magnitude of the effect of causal polymorphism in the estimation of these intervals, which is well established for linkage mapping [47], should be explored further. Other approaches have been proposed to cluster SNPs according to LD [48, 49]. These approaches aim at segmenting the genome in different haplotype blocks separating by high recombination regions. These methods are difficult to use for estimating putative windows inside which the causal polymorphisms are located because such approaches are not centered on the associated SNP.

Several QTLs identified by $L D \_$win in our study correspond to regions previously identified: in particular, six 
regions associated with female flowering time [26] and 30 regions associated with different traits in the Cornfed dent panel [11]. Conversely, we did not identify in our study any QTL associated to the florigen $Z C N 8$, which showed significant effect in these two previous studies. One of the explanation is that we narrowed the flowering time range in our study, in particular by eliminating early lines. This reduced the representation of the early allele at the $Z \mathrm{cn} 8$ locus, leading to a MAF of 0.27 in our study vs 0.35 in Rincent et al. [11], which can slightly diminish the power of the tests [14]. Also, this effect may have strengthened by frequency evolution at loci involved in epistatic interactions with Zcn8 (see [50] for a recent demonstration of such effects).

\section{Complementarity of $600 \mathrm{~K}$ and GBS for QTL detection resulted mostly from the tagging of different haplotypes rather than the coverage of different genomic regions} Number of significant SNPs and QTLs increased with the increase in marker number (Table 4, Additional file 9: Figure S9). This could be explained partly by a better coverage of some genomic regions by SNPs, notably in high recombinogenic regions which showed a very short LD extent and were enriched in QTLs (Additional file 16: Table S3). Numerous new QTLs identified by the $600 \mathrm{~K}$ and GBS as compared with those identified by the $50 \mathrm{~K}$ were detected in high recombinogenic regions that were considerably less covered by the $50 \mathrm{~K}$ than the $600 \mathrm{~K}$ or GBS (Fig. 1 and Additional file 3: Figure S3).

The high complementarity for QTL detection between GBS and $600 \mathrm{~K}$ was only explained to a limited extent by the difference of the SNP distribution and density along the genome, since these two technologies targeted similar regions as showed by the coverage analysis (Fig. 1 and Additional file 3: Figure S3). However, at a finer scale, SNPs from the $600 \mathrm{~K}$ and GBS could tag close, but different genomic regions around genes. SNPs from the $600 \mathrm{~K}$ were mostly selected within coding regions of genes [4], whereas SNPs from GBS targeted more largely low copy regions, which included coding but also regulatory regions of genes [31, 37]. To further analyse the complementarity of the technologies, we analysed local haplotypes and the effect of genome coverage differences between technologies on QTL detection. We showed that both technologies captured different haplotypes when similar genomic regions were targeted (Fig. 5). In this figure, two QTLs were specifically detected by markers from either $600 \mathrm{~K}$ or GBS, although there are several markers from the other technology very close from the most associated marker, considering the size of LD windows around it. Additionally, we did not observe an enrichment of QTLs specifically detected by one technology in $20 \mathrm{kbp}$-intervals with high genomic coverage difference between $600 \mathrm{~K}$ and GBS. Hence, we pinpointed that GBS and DNA arrays are highly complementary for QTL detection because they tagged different haplotypes rather than different regions (Fig. 5). Based on the L-shaped MAF distribution, which suggests no ascertainment bias, and the high number of sequenced lines used for the GBS, we expect a closer representation of the variation present in our panel by this technology compared to the $600 \mathrm{~K}$, but this comes to the cost of an enrichment in rare alleles. Both factors tend to counterbalance each other in terms of GWAS power (Additional file 13: Figure S12).

Our results suggest that we did not reach saturation with our c. 800,000 SNPs because (i) some haplotypes certainly remain not tagged (ii) the genome coverage was not complete, and (iii) the number of significant SNPs and QTLs continued to increase with marker density (Additional file 9: Figure S9). Considering LD and marker density, the genotypic data presently available were most likely enough to well represent polymorphisms in the centromeric regions, whereas using more markers would be beneficial for telomeric regions. New approaches based on resequencing of representative lines and imputation are currently developed to achieve this goal.

\section{Methods}

\section{Plant material and phenotypic data}

The panel involves 247 maize inbred lines, further referred to as DROPS panel (Additional file 17: Table S4). They include 164 lines from a wider panel of lines from Europe and America [11] and 83 additional lines derived from public breeding programs in Hungary, Italy and Spain and recent lines free of patent from the USA. All lines belong to the dent genetic group, which can be subdivided in different sub-groups (see [11, 29]). Lines were selected within a restricted flowering time window (10 days) in order to limit the effect of drought escape due to flowering time variation in the identification of genomic regions involved in drought tolerance [29]. Candidate lines with poor sample quality, i.e. high level of heterozygosity, or high relatedness with other lines were discarded in this selection. The lines selection was also guided by pedigree to avoid as far as possible overrepresentation of some ancestral materials.

The 247 inbred lines were all crossed with a common line (UH007) from the Flint genetic groups to obtain 247 hybrids (hybrid panel). Dent and Flint genetic groups are known to be complementary to produce hybrids [51]. Further, as UH007 is unrelated to any line in the panel, no hybrid is affected by inbreeding depression. This guarantees that hybrids 
have a level of performance and an overall physiology comparable to that of varieties used in agriculture. Conversely, field evaluation of inbred lines per se would have diminished yield by more than $50 \%$.

Experimental design and model used for obtaining adjusted means for male flowering time (Day To Anthesis, DTA), plant height (plantHT), and grain yield (GY) were previously described [29]. While DTA and GY were previously analyzed in [29], PlantHT was not. Briefly, the hybrid panel were evaluated for these three traits in 22 experiments (combination year $\mathrm{x}$ site $\mathrm{x}$ water regime), i.e. at seven sites in Europe, during two years (2012 and 2013), and for two water treatments (watered and rainfed) [29]. Experiments were designed as alpha-lattice designs with two and three replicates for watered and rain-fed regimes, respectively. Grain yield $\left(\mathrm{tha}^{-1}\right)$ was adjusted to $15 \%$ moisture. The adjusted mean (Best Linear Unbiased Estimation, BLUEs, https://doi.org/1 $0.15454 /$ IASSTN) of the three traits were estimated per environment (site $\times$ year $\times$ water regime) using a mixed model based on fixed hybrid and replicate effects, random spatial effects (rows and columns), and spatially correlated errors in order to take into account spatial variation of micro-environment in each field trial (see [29] for more details). The same model, but with random hybrids effects, was used to estimate variance components. Models were fitted with ASReml-R [52]. Narrow-sense heritability of each trait in each environment were also estimated as in [29] (Additional file 18: Table S5). As all hybrids share a common parent (UH007), adjusted means (BLUEs) of hybrids were combined with genotyping data of the corresponding dent inbred lines of the panel to perform GWAS, following a usual practice in maize genetics [11].

\section{Genotyping and genotyping-by-sequencing data}

The 247 inbred lines were genotyped using three technologies: a maize Illumina Infinium HD $50 \mathrm{~K}$ array [3], a maize Affymetrix Axiom $600 \mathrm{~K}$ array [4], and Genotyping-By-Sequencing [2, 37]. In the arrays, DNA fragments are hybridized with probes attached to the array (Additional file 19: Notes S1 for the description of the data from the two SNP-arrays). Genotyping-bysequencing technology is based on multiplex resequencing of tagged DNA using restriction enzyme (Keygene N.V. owns patents and patent applications protecting its Sequence Based Genotyping technologies) [2]. Cornell Institute (NY, USA) processed raw sequence data using a multi-step Discovery and a one-step Production pipeline (TASSEL-GBS) in order to obtain genotypes (Additional file 19: Notes S1). An imputation step of missing genotypes was carried out by Cornell Institute [38], which utilized an algorithm that searches for the closest neighbour in small SNP windows across the haplotype library [37].

We applied different filters (heterozygosity rate, missing data rate, minor allele frequency) for a quality control of the genetic data before performing the diversity and association genetic analyses. For GBS data, the filters were applied after imputation using the method "Compilation of Cornell homozygous genotypes and Beagle genotypes" ( $\mathrm{GBS}_{5}$ in Additional file 1: Figure S1; See section "Evaluating Genotyping and Imputation Quality"). We eliminated markers that had an average heterozygosity and missing data rate higher than 0.15 and 0.20 , respectively, and a Minor Allele Frequency (MAF) lower than 0.01 for the diversity analyses and 0.05 for the GWAS (Additional file 20: Table S6). Individuals which had heterozygosity and/or missing data rate higher than 0.06 and 0.10 , respectively, were eliminated. Filtered imputed genotyping data for $50 \mathrm{~K}, 600 \mathrm{~K}$ and GBS were available at https://doi.org/10.15454/ AEC4BN.

\section{Evaluating genotyping and imputation quality}

Estimation of genotyping and imputation quality was performed using the entire panel except two inbred lines that had different seedlots between technologies. The 50 $\mathrm{K}$ and the $600 \mathrm{~K}$ were taken as reference to compare the concordance of genotyping (genotype matches) with the imputation of GBS based on their position. While SNP positions and orientation from GBS were called on the reference maize genome B73 AGP_v2 (release 5a) [53], flanking sequences of SNPs in the $50 \mathrm{~K}$ were primary aligned on the first maize genome reference assembly B73 AGP_v1 (release 4a.53) [54]. Both position and orientation scaffold carrying SNPs from the $50 \mathrm{~K}$ can be different in the AGP_v2, which could impair correct comparison of genotype between the $50 \mathrm{~K}$ and GBS. Hence, we aligned flanking sequences of SNPs from the $50 \mathrm{~K}$ on maize B73 AGP_v2 using the Basic Local Alignment Search Tool (BLAST) to retrieve both positions and genotype in the same and correct strand orientation (forward) to compare genotyping. The number of common markers between the $50 \mathrm{~K} / 600 \mathrm{~K}, 50 \mathrm{~K} / \mathrm{GBS}$, GBS/ $600 \mathrm{~K}$ and $50 \mathrm{~K} / 600 \mathrm{~K} / \mathrm{GBS}$ was 36,395, 7,018, 25,572 and 5,947 SNPs, respectively. The comparison of the genotyping and imputation quality between the $50 \mathrm{~K} /$ GBS, $50 \mathrm{~K} / 600 \mathrm{~K}$ and $600 \mathrm{~K} / \mathrm{GBS}$ was done on 5,336 and 24,286 and 26,154 common markers, respectively. The comparison for the $50 \mathrm{~K}$ involved PANZEA markers, prefixed as "PZE" [55]. In order to achieve these comparisons, we considered the direct reads from GBS $\left(G_{B S}\right)$ and four approaches for imputation $\left(\mathrm{GBS}_{2}\right.$ to $\mathrm{GBS}_{5}$, Additional file 1: Figure S1). $\mathrm{GBS}_{2}$ approach consisted of one imputation step from the direct read by Cornell University, using TASSEL software, but missing 
data was still present. $\mathrm{GBS}_{3}$ approach consisted of imputation by Beagle v3 [56] of the missing data of $\mathrm{GBS}_{1}$. To compare data from $\mathrm{GBS}_{3}$ and $\mathrm{GBS}_{2}$ to those of the $50 \mathrm{~K}$ and $600 \mathrm{~K}$, missing data in $\mathrm{GBS}_{2}$ were excluded from $\mathrm{GBS}_{3}$. In $\mathrm{GBS}_{4}$, genotype imputation by Beagle was performed on Cornell imputed data after replacing the heterozygous genotypes with missing data. $\mathrm{GBS}_{5}$, consisted of homozygous genotypes of $\mathrm{GBS}_{2}$ completed by values imputed in $\mathrm{GBS}_{3}$, no missing data remained (Additional file 1: Figure S1).

\section{Diversity analyses}

After excluding the unplaced SNPs and applying the filtering criteria for the diversity analyses (MAF > 0.01), we obtained the final genotyping data of the 247 lines with 44,729 SNPs from the $50 \mathrm{~K}$, 506,662 SNPs from the $600 \mathrm{~K}$ array, and 395,024 SNPs from the GBS (Additional file 20: Table S6). All markers of the $600 \mathrm{~K}$ and $\mathrm{GBS}_{5}$ that passed the quality control were used to perform the diversity analyses (estimation of $\mathrm{Q}$ genetic groups and $\mathrm{K}$ kinships). For the 50 $\mathrm{K}$, we used only the PANZEA markers (29,257 SNPs) [55] in order to reduce the ascertainment bias noted by Ganal et al. [3] when estimating Nei's index of diversity [57] and relationship coefficients. Genotypic data generated by the three technologies were organized as $G$ matrices with $N$ rows and $L$ columns, $N$ and $L$ being the panel size and number of markers, respectively. Genotype of individual $i$ at marker $l$ $\left(G_{i, l}\right)$ was coded as 0 (the homozygote for an arbitrarily chosen allele), 0.5 (heterozygote), or 1 (the other homozygote). Identity-By-Descent (IBD) was estimated according to Astle and Balding [19]:

$$
\operatorname{K}_{\text {Freq }}{ }_{i, j}=\frac{1}{L} \sum_{l=1}^{L} \frac{\left(G_{i, l}-p_{l}\right)\left(G_{j, l}-p_{l}\right)}{p_{l}\left(1-p_{l}\right)}
$$

where $p_{l}$ is the frequency of the allele coded 1 of marker $l$ in the panel of interest, $i$ and $j$ indicate the inbred lines for which the kinship was estimated. We also estimated the Identity-By-State (IBS) by estimating the proportion of shared alleles. For GWAS, we used $K_{-}$ Chr [13] that are computed using similar formula as $K \_F r e q$, but with the genotype data of all the chromosomes except the chromosome of the SNP tested. This formula provides an unbiased estimate of the kinship coefficient and weights by allelic frequency assuming Hardy-Weinberg equilibrium. Hence, relatedness is higher if two individuals share rare alleles than common alleles.

Genetic structure was analysed using the sofware $A D$ MIXTURE v1.22 [17] with a number of groups varying from 2 to 10 for the three technologies. We compared assignation by ADMIXTURE of inbred lines between the three technologies by estimating the proportion of inbred lines consistently assigned between technologies two by two ( $50 \mathrm{~K}_{\text {vs }} \mathrm{GBS}_{5}, 50 \mathrm{~K}$ vs $600 \mathrm{~K}, 600 \mathrm{~K}_{\text {vs }} \mathrm{GBS}_{5}$ ) using a threshold of 0.5 for admixture.

Expected heterozygosity (He) [57] was estimated at each marker as $2 p_{l}\left(1-p_{\nu}\right)$ and was averaged on all the markers for a global characterization of the panel for the three technologies. Principal Coordinate Analyses ( $\mathrm{PCoA})$ were performed on the genetic distance matrices [58], estimated as $1_{N, N}-K_{-}$Freq, where $1_{N, N}$ is a matrix of ones of the same size as $K_{-}$Freq.

\section{Linkage disequilibrium analyses}

We first analyzed the effect of the genetic structure and kinship on linkage disequilibrium (LD) extent within and between chromosomes by estimating genome-wide linkage disequilibrium using the 29,257 PANZEA SNPs from the $50 \mathrm{~K}$. Four estimates of LD were used: the squared correlation $\left(r^{2}\right)$ between allelic dose at two markers [59], the squared correlation taking into account global kinship with $K \_$Freq estimator $\left(r^{2} K\right)$, the squared correlation taking into account population structure $\left(r^{2} S\right)$, and the squared correlation taking into account both $\left(r^{2} K S\right)$ [16].

To explore the variation of LD decay and the stability of LD extent along the chromosomes, we estimated LD between a non-redundant set of 810,580 loci from the GBS, the $50 \mathrm{~K}$ and $600 \mathrm{~K}$. To save computation time, we calculated LD between loci within a sliding window of 1 cM. Genetic position was obtained by projecting the physical position of each locus using a smooth.spline function $\mathrm{R}$ calibrated on the genetic consensus map of the Cornfed Dent Nested Association Mapping (NAM) design [42]. We used the estimator $r^{2}$ and $r^{2} K$ using 10 different kinships $K_{-} C h r$. This last estimator was calculated because it corresponds exactly to the LD used to map QTL in our GWAS model. It determines the power of GWAS to detect QTL considering that causal polymorphisms were in LD with some polymorphisms genotyped in our panel [16]. To study LD extent variation, we estimated LD extent by adjusting Hill and Weir's model [39] using non-linear regression (nls function in R-package nlme) against both physical and genetic position within each chromosome. Since recombination rate $(\mathrm{cM} / \mathrm{Mbp})$ varied strongly along the genome (Fig. 1 and Additional file 3: Figure S3), we defined high $(>0.5 \mathrm{cM} /$ $\mathrm{Mbp})$ and low $(<0.5 \mathrm{cM} / \mathrm{Mbp})$ recombinogenic genomic regions within each chromosome. We adjusted Hill and Weir's model [39] separately in low and high recombinogenic regions (Additional file 16: Table S3) by randomly sampling 100 sets of 500,000 pairs of loci distant from less than $1 \mathrm{cM}$. This random sampling avoided overrepresentation of pairs of loci from low recombinogenic regions due to the sliding-window approach (Fig. 3). 
500,000 pairs of loci represented 0.36\% (Chromosome 3 /High rec) to $1.20 \%$ of all pairs of loci (Chromosome $8 /$ High rec).

For all analyses, we estimated LD extent by calculating the genetic and physical distance for the fitted curve of Hill and Weir's Model that reached $r^{2} K=0.1, r^{2} K=0.2$ and $r^{2} K=0.4$

\section{Genome coverage estimation}

In order to estimate the genomic regions in which the effect of an underlying causal polymorphisms could be captured by GWAS using LD with SNP from three technologies, we developed an approach to define LD windows around each SNP with MAF $\geq 5 \%$ based on LD extent (Fig. 3). To set the LD window around each SNP, we used $\mathrm{LD}$ extent with $r^{2} K=0.1$ (negligible LD), $r^{2} K=0.2$ (intermediate LD) and $r^{2} K=0.4$ (high LD) estimated in low and high recombinogenic regions for each chromosome. We used the global LD decay estimated for these large chromosomal regions rather than local LD extent (i) to avoid bias due to SNP sampling within small genomic regions, (ii) to reduce computational time, and (iii) to limit the impact of possible local error in genome assembly. In low recombinogenic regions, we used the physical LD extent, hypothesizing that recombination rate is constant along physical distance in these regions. In high recombinogenic regions, we used the genetic LD extent since there is a strong variation of recombination rate by base pair along the physical position (Fig. 1 and Additional file 3: Figure S3). We then converted genetic LD windows into physical windows by projecting the genetic positions on the physical map using the smooth.spline function implemented in $\mathrm{R}$, calibrated on the NAM dent consensus map [42]. Reciprocally, we obtained the genetic positions of LD windows in low recombinogenic regions by projecting the physical boundaries of $\mathrm{LD}$ windows on the genetic map.

To estimate coverage of the three technologies to detect QTLs based on their SNP distribution and density, we calculated cumulative genetic and physical lengths that are covered by LD windows around the markers, considering different LD extents for each chromosome $\left(r^{2} K=0.1\right.$, $\left.r^{2} K=0.2, r^{2} K=0.4\right)$. In order to explore variation of genome coverage along the chromosome, we estimated the proportion of genome covered using a sliding-windows approach based on variable physical distances $(20,100$, 500, $2000 \mathrm{kbp}$ ) considering LD extent for a $r^{2} K=0.1$.

\section{Statistical models for association mapping}

We used four models to determine the statistical models that control best the confounding factors (i.e. population structure and relatedness) in GWAS (Additional file 21: Notes S2). We tested different software implementing either approximate (EMMAX) [8] or exact computation of standard test statistics (ASReml and FaST-LMM) [6, 52] for computational time and GWAS results differences. Single-trait, single-environment GWAS was performed for each marker for each environment and all traits using FaST-LMM. We selected the mixed model using K_Chr, estimated from PANZEA markers of the $50 \mathrm{~K}$ to perform GWAS on 66 situations (environment $\times$ trait) (Additional file 21: Notes S2). We developed a GWAS pipeline in $R v 3.2 .1$ [60] calling FaST-LMM software and implementing [13] approaches to conduct single trait and single environment association tests.

To take into account multiple tests in GWAS and their dependence, we applied the methods of Moskvina and Schmidt [61] and Gao et al. [62,63] to infer the number of independent tests to be considered in the Bonferroni formula. Using the Gao et al. [62, 63] approaches, we estimated the number of independent tests for GWAS at 15,780 for the $50 \mathrm{~K}, 92,752$ for the $600 \mathrm{~K}$, 109,117 for the $\mathrm{GBS}_{5}$ and 191,026 for the combined genetic data (i.e. merging of $50 \mathrm{~K}, 600 \mathrm{~K}, \mathrm{GBS}$ ), leading to different $-\log _{10}(p$-value $)$ thresholds: $5.49,6.27,6.34$ and 6.58 , respectively. Because of these differences, we used two thresholds of $-\log _{10}(p$-value $)=5$ (less stringent) and 8 (hightly conservative and slightly above Bonferroni) for comparing GWAS to avoid the differences of identification of significant SNPs between the technologies due to the choice of the threshold.

\section{Methods for grouping associated SNPs into QTLs}

We used two approaches based on LD for grouping significant SNPs. The first approach $\left(L D_{-}\right.$win) used LD windows, previously described, to group significant SNPs into QTLs considering that all significant SNPs with overlapping LD windows of $r^{2} K=0.1$ belong to the same QTL (Fig. 3). We hypothesized that significant SNPs with overlapping LD windows at $r^{2} K=0.1$ captured the same causal polymorphism and were therefore a single and unique QTL. For the second approach (LD_adj), significant SNPs were grouped into a same QTL if they were connected in terms of LD $\left(r^{2} K\right.$ between adjacent significant SNPs superior to 0.5 ). We used LD heatmaps for comparing the SNP grouping produced by the two approaches on the three different traits across all environments (Additional file 7: Figure S7-LD-Adjacent and Additional file 8: Figure S8-LD-Windows). All scripts are implemented in R software [60]. Scripts to group associated SNPs into QTLs based on two LD approaches ( $L D \_$win and $\left.L D \_a d j\right)$ are available on request.

\section{Resampling approach to analyse the effects of MAF distribution, SNP distribution along the genome and SNP density on QTL detection}

To study the effects of SNP density, MAF distribution and SNP distribution along the genome on association 
and QTL detection, we used a resampling approach of several sets of SNPs displaying different MAF distribution and SNP distribution along the chromosome. We compared these modalities with different SNP densities (50,000, 100,000, 150,000, 200,000, 250,000 markers). In this resampling approach, we considered all markers together and that both associations and QTLs detected by the whole SNP sets are true. We selected only markers having MAF above 5\%. To study the effect of MAF distribution on QTL detection, SNPs were classified in 5 MAF classes $(0-0.1,0.1-0.2,0.2-0.3,0.3-0.4$ and $0.4-$ 0.5 ) and SNPs were randomly selected in each classes according to the MAF distributions: 1) similar to GBS (GBS_MAF), 2) similar to $600 \mathrm{~K}$ (600 K_MAF), 3) with equal frequency for the five MAF classes (Flat_MAF), 4) skewed towards high MAF (High_MAF) with SNP frequency of $0,0,0.2,0.4,0.4$ in $(0-0.1],(0.1-0.2],(0.2-$ 0.3], (0.3-0.4], (0.4-0.5] MAF classes, respectively, and 5) skewed towards low MAF (Low_MAF) with SNP frequency of $0,0,0.2,0.4,0.4$ in $(0-0.1],(0.1-0.2],(0.2-$ $0.3],(0.3-0.4],(0.4-0.5]$ MAF classes, respectively.

To study the effect of SNP distribution along the genome on QTL detection, we compared five different SNP distributions along the chromosome: 1) evenly distributed according to the physical distance (Dens_Phys), 2) evenly distributed according to the genetic distance (Dens_Gen), 3) distributed like GBS (Dens_GBS), 4) distributed similarly to $600 \mathrm{~K}$ (Dens_600 K), and 5) distributed like $50 \mathrm{~K}$ (Dens_50 K). SNPs were sampled randomly according to the different densities in contiguous windows of $10 \mathrm{Mbp}$.

\section{Additional files}

Additional file 1: Figure S1. Different approaches used to impute missing data of the GBS. We considered the direct reads from $\mathrm{GBS}\left(\mathrm{GBS}_{1}\right)$ and four approaches for imputation $\left(\mathrm{GBS}_{2}\right.$ to $\left.\mathrm{GBS}_{5}\right)$. $\mathrm{GBS}_{2}$ approach consisted in one imputation step from the direct read by Cornell University, using TASSEL software, but missing data was still present. $\mathrm{GBS}_{3}$ approach consisted in a genotype imputation of the whole missing data of the direct read by Beagle v3. In $\mathrm{GBS}_{4}$, genotype imputation by Beagle was performed on Cornell imputed data after replacing the heterozygous genotypes into missing data. $\mathrm{GBS}_{5}$, consisted in homozygous genotypes of $\mathrm{GBS}_{2}$ completed by values imputed in $\mathrm{GBS}_{3}$. (DOCX $14 \mathrm{~kb}$ )

Additional file 2: Figure S2. Comparison of genotyping data between $50 \mathrm{~K}$ and $600 \mathrm{~K}$ arrays, and GBS. (a) Distribution of minor allele frequency per SNP before filtering (monomorphic SNPs removed). (b) Distribution of SNP missing data proportion for the 50K array, 600K array, GBS direct reads $\left(\mathrm{GBS}_{1}\right)$ and $\mathrm{GBS}$ after imputation by Cornell Institute $\left(\mathrm{GBS}_{2}\right.$, note that the scale of the $x$-axes is different). (c) Relatedness distribution (Identity-By-State, IBS) after QC filtering with MAF $\geq 1 \%$ (IBS using GBS, was not estimated because of the low calling rate). (DOCX $71 \mathrm{~kb}$ )

Additional file 3: Figure S3. Variation of the markers density, the recombination rate and the genome coverage in non-overlapping $2 \mathrm{Mbp}$ windows along each chromosome except chromosome 3 (presented in Fig. 1). Markers have MAF above $5 \%$. Top panel shows the variation of SNP number. In the bottom panel, dotted line represents the variation of recombination rate (cM / Mbp) and solid lines the proportion of genome covered by the SNPs using the cumulated length of physical LD windows around each SNP in each 2Mbp-windows. In these two panel, green, blue, red and black lines represent variation for GBS, 600K, 50K and combined technologies, respectively. Vertical dotted gray lines indicate limits of centromeric regions. Vertical lines between the two panels indicate the position of QTLs for flowering time (DTA), grain yield (GY) and Plant Height (PHT). Green, blue, red vertical lines indicate QTLs detected only by GBS, 600K and 50K technologies, respectively. Grey vertical lines indicate QTL detected by at least two technologies. Only QTL including a marker associated with $-\log _{10}$ (pval) above 6 were shown. (PDF $152 \mathrm{~kb}$ )

Additional file 4: Figure S4. Contribution of four ancestral populations to 247 inbred lines after ADMIXTURE analysis. Markers from the 50K (top), $600 \mathrm{~K}$ (middle) and GBS (bottom) were used. One vertical bar corresponds to one individual. Lines were ordered according to contributions observed for the 50K. From left to right, we have Stiff Stalk lines type B73 and B14a (blue), lodent lines type PH207 (red), Lancaster lines type Mo17 and Oh43 (turquoise), a group of lines assembling W117, F7057 type lines (green). (DOCX $239 \mathrm{~kb}$ )

Additional file 5: Figure S5. Correlation between kinship matrix estimated by different technologies. Correlation $(r)$ between the IBS and IBD (K_Freq) for each technology (A). Correlation of IBD (B) and IBS (D) between the three technologies (after imputation). (C) Correlation of IBD between the three technologies after removing the excess of rare alleles in the GBS to have the same distribution of MAF as in the 50K and the 600K. The red line is the bisector. (DOCX $255 \mathrm{~kb}$ )

Additional file 6: Figure S6. Heatmaps of genome-wide linkage disequilibrium (LD) between all markers within and between chromosomes using PANZEA SNPs from the 50K. All SNPs were ordered according to their position on the genome. "Unpl" after chromosome 10 refers to unplaced SNPS, in an arbitrary order. Dots represented LD between two loci and were colored according to their strength. Classical LD measurement $r^{2}$ between loci were represented within triangle below the diagonal. Linkage disequilibrium corrected for structure $\left(r^{2} S, A\right)$, relatedness $\left(r^{2} K, B\right)$ or both $\left(r^{2} K S, C\right)$ were represented within triangle above the diagonal. (DOCX $442 \mathrm{~kb}$ )

Additional file 7: Figure S7. QTL limits obtained by the LD_adj approach projected on heatmaps representing the level of LD between associated SNPs for each trait (DTA: male flowering time, plantHT: plant height and GY: grain yield) and each chromosome. Upper and lower triangles on the heatmaps represent the $r^{2}$ and $r^{2} K$ values between associated SNPs, respectively. Linkage disequilibrium between loci was colored according to values from weak LD (yellow) to high LD (red). The significant markers were ordered according to their physical positions on the chromosome and were represented by ticks on the four sides of the heatmaps. Limits of QTLs were displayed by gray dotted lines. QTL numbers were indicated in gray on the top and the right of each heatmap. (PDF $7373 \mathrm{~kb}$ )

Additional file 8: Figure S8. QTL limits obtained by the LD_win approach projected on heatmaps representing the level of LD between associated SNPs for each trait (DTA: male flowering time, plantHT: plant height and GY: grain yield) and each chromosome. Upper and lower triangles on the heatmaps represented the $r^{2}$ and $r^{2} K$ values between associated SNPs, respectively. Linkage disequilibrium between loci was colored according to values from weak LD (yellow) to high LD (red). The significant markers were ordered according to their physical positions on the chromosome and were represented by ticks on the four sides of the heatmaps. Limits of QTLs were displayed by gray dotted lines. QTL numbers were indicated in gray on the top and the right of each heatmap. (PDF $7360 \mathrm{~kb}$ )

Additional file 9: Figure S9. Number of significant SNPs (blue line) and QTLs (red line) identified as a function of SNP density ( $x$-axis) for the male flowering time (DTA), plant height (PlantHT) and grain yield (GY). (DOCX $125 \mathrm{~kb}$ )

Additional file 10: Table S1. Summary of all the QTLs identified for the male flowering time (DTA), plant height (plantHT) and grain yield (GY). "LowerLimit" and "UpperLimit" columns are the lower and upper physical limits for each QTL. The "Rec" column indicates if the QTL is located in a high or low region of recombination. "NbSNP50", "LogPvaMax50", "NbSNP600", "LogPvaMax600", "NbSNPGBS", "LogPvaMaxGBS" are the number of significant SNPs and the most significant $-\log _{10}($ Pval) within the QTL for each technology across all environments. The physical 
position ("PosMax"), the proportion of the variance explained ("R2_LDMax") and the effect ("EffectMax") of the most significant SNP within the QTL is shown. "NbDiffEnv" gives the number of different situations that detected the QTL. (CSV $50 \mathrm{~kb}$ )

\section{Additional file 11: Figure S10. Examples of QTL detection on} Chromosome 3, 6 and 8 for the different traits. The top panel represents the distribution of the QTLs along the chromosome of interest, for the different technologies. The vertical red line in this panel localizes the SNP chosen as reference for the QTL (marker with the strongest association). The middle panel is a zoom in the vicinity of the reference SNP, showing the Local distribution of the - $\log _{10}(p$-value). The bottom panel is the same zoom as the middle panel and shows the local linkage disequilibrium corrected by the kinship $\left(r^{2} k\right)$ of all SNPS, within this region, within the reference SNP. Ticks on different $x$-axes show the marker density of the three technologies (red for the 50K, blue for the 600K and green for the GBS, black for all markers). (DOCX $1632 \mathrm{~kb}$ )

Additional File 12: Figure S11. Effect of minor allelic frequency distribution, SNP distributions along the genome and SNP densities on the number of associated SNP and QTL detected. Boxplot were drawn on 100 sets of 50000 to 250000 markers sampled according to different MAF distributions (A, B) and different SNP distributions along the genome (C, D). A, C: number of SNP associated; B, D: Number of QTL detected. In A and B, 600K_MAF (yellow), GBS_MAF (green), Low_MAF (cyan), Flat_MAF (blue), High_MAF (pink) on $\mathrm{x}$ axis indicate boxplots corresponding to MAF distribution similar to $600 \mathrm{~K}$, similar to GBS, skewed towards low MAF, flat MAF and skewed toward high MAF, respectively. In C and D, Dens_50K (red), Dens_600K (yellow), Dens_GBS (cyan), Dens_Gen (blue), Dens_Phys (pink) on $x$ axis indicate distribution of SNPs along the genome corresponding to $50 \mathrm{~K}, \mathrm{GBS}, 600 \mathrm{~K}$, even genetic and physical distances, respectively. For $A, B, C$ and $D$, modalities indicated as "Random" in $\mathrm{x}$ axis correspond to random sample of SNP. Number of markers for each boxplot are indicated after the point. (PDF $281 \mathrm{~kb})$

Additional file 13: Figure S12. Distribution of markers, associations and QTLs according to the MAF classes for 50K, 600K GBS, and ALL technologies. A) Number of markers, B) Proportion of markers, C) Proportion of Association, D) Proportion of QTLs. (PDF $32 \mathrm{~kb}$ )

Additional file 14: Figure S13. Colocalization of QTLs between the traits. Number of QTLs specific and shared by the three traits across all environments. Note that several QTLs from one trait were sometimes included in a single QTL of another trait. (DOCX $48 \mathrm{~kb}$ )

Additional file 15: Table S2. Stability of QTLs across environments for the male flowering time (DTA), Plant Height (PlantHT), Grain Yield (GY)

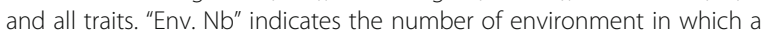
QTL was detected. Next four columns indicate the number of QTL corresponding to each category. (DOCX $14 \mathrm{~kb}$ )

Additional file 16: Table S3. Proportion of low and high recombination regions, recombination rate and percentage of QTLS located in these regions for the three traits. "Chr" indicates the chromosome. Physical and genetic size columns indicated the size of each chromosome in $\mathrm{bp}$ and $\mathrm{cM}$, respectively. Average recombination rate ("RecRate") and proportion of the physical ("Phys") and genetic ("Genetic) map in high recombination regions ("HighRec", >0.5 cM / Mbp) for each chromosome are shown. Percentage of QTL in high recombination regions were displayed for three traits (DTA: male flowering, PlantHT: Plant Height, GY: Grain Yield). (DOCX 16 kb)

Additional file 17: Table S4. Description of inbred lines. Variety and accession along with the breeders, seeds providers and genetic groups obtained using ADMIXTURE for K=4 (Stiff Stalk, lodent, Lancaster, Other). (CSV $260 \mathrm{~kb}$ )

Additional file 18: Table S5. Narrow sense heritability $\left(h^{2}\right)$ and variance components $\left(V_{g}\right.$, genetic variance; $V_{e}$, residual variance). The heritability and variance components were estimated for all traits (grain yield, male flowering time and plant height) using the R package Heritability [1]. (DOCX $18 \mathrm{~kb}$ )

Additional file 19: Notes S1. Differences between SNP-arrays and GBS discrovery / pipelines. (DOCX $15 \mathrm{~kb}$ )

Additional file 20: Table S6. Number of SNPs called, after OC filtering (MAF>1\%) and useful for GWAS (MAF $\geq 5 \%$ ). Note that GBS1 have SNPs with
100\% missing genotypes which were removed while GBS2 used external haplotype library which allow to impute loci with 100\% missing data. It conducted to a smaller number of SNPs for GBS1 than GBS2. (XLS $33 \mathrm{~kb}$ )

Additional file 21: Notes S2. GWAS statistical models and effects of confounding factors on GWAS. (DOCX $14 \mathrm{~kb}$ )

\section{Abbreviations}

DTA: Day to Anthesis; GBS: GenotypingBySequencing; GWAS: Genome-Wide Association Studies; GY: Grain yield adjusted at 15\% moisture; HRR: High recombinogenic regions; LD: Linkage disequilibrium; LRR: Low recombinogenic regions; MAF: Minimum allelic frequency; plantHT: Plant height; QTL: Quantitative Trait Locus; SNP: Single Nucleotide Polymorphism

\section{Acknowledgements}

We are grateful to key partners from the field: Pierre Dubreuil, Cécile Richard, Jérémy Lopez (Biogemma), Tamás Spitkó (MTA ATK), Therese Welz (KWS), Franco Tanzi, Ferenc Racz, Vincent Schlegel (Syngenta) and Maria Angela Canè (UNIBO). We also acknowledge Björn Usadel and Axel Nagel (MPI) for data management. We thank Willem Kruijer, Fred Van Eeuwijk (WUR), Tristan Mary-Huard and Laurence Moreau (INRA) for helpful discussions and statistical advice. We are grateful to Chris-Carolin Schön (TUM) for providing an early access to the Affymetrix Axiom $600 \mathrm{~K}$ array and Edward Buckler (USDA) for providing genotyping using GBS. We are also grateful to partners of the CornFed project, Univ. Hohenheim (Germany), CSIC (Spain), CRAG (Spain), MTA ATK (Hungary), NCRPIS (USA), CRB Maize (France) and CRA-MAC (Italy) who contributed to the genetic material. We are grateful to Matthieu Falque for providing consensus genetic map. We acknowledge three reviewers for their helpful comments.

\section{Authors' contributions}

SSN, SDN and AC, designed the studied and wrote the article. SSN performed genotyping data quality control, imputation and genetic analyses. SDN developed and performed LD analyses. AC designed the association panel with the help of SDN and CW. CB participated in assembling the dent inbred lines panel, organizing the germplasms and field work for seeds production. EJM, CW and FT collected and analysed the phenotypic data. VC and DM performed DNA extraction and prepared the samples. All authors critically reviewed and approved the final manuscript.

\section{Funding}

This project (Project ID: 244374) was funded under the European FP7- KBBE (CP - IP - Large-scale integrating project, DROPS) and the Agence Nationale de la Recherche project ANR-10-BTBR-01 (ANR-PIA AMAIZING). DROPS and AMAMIZNG funded 4 year's postdoctoral fellowship of Sandra Negro. Design of panel, 20 field experiments, DNA extraction, phenotyping analysis and the genotyping of panel by $600 \mathrm{~K}$ array as well as GWAS analysis were supported by DROPS projects. 2 field experiments, genotyping of panel by GBS, development of methods based on Linkage disequilibrium analysis and imputation were supported by AMAIZING. Interpretation of data and writing the manuscript were done in the framework of AMAIZNG and DROPS projects.

\section{Availability of data and materials}

All the genotyping data used in this study can be found at https://doi.org/1 0.15454 /AEC4BN.

The GWAS results can be found at https://doi.org/10.15454/6TL2N4 The phenotypic dataset can be found at https://doi.org/10.15454/IASSTN.

Ethics approval and consent to participate

Not applicable.

\section{Consent for publication}

Not applicable.

\section{Competing interests}

The authors declare that they have no competing interests.

\section{Author details}

${ }^{1}$ GQE - Le Moulon, INRA, Univ. Paris-Sud, CNRS, AgroParisTech, Université Paris-Saclay, 91190 Gif-sur-Yvette, France. 'Laboratoire d'Ecophysiologie des 
Plantes sous Stress Environnementaux (LEPSE), UMR759, INRA, SupAgro, 34060 Montpellier, France. ${ }^{3}$ Present address: Biometris, Department of Plant Science, Wageningen University and Research, 6700 AA Wageningen, The Netherlands.

\section{Received: 5 February 2019 Accepted: 5 July 2019}

Published online: 16 July 2019

\section{References}

1. Tian F, Bradbury PJ, Brown PJ, Hung H, Sun Q, Flint-Garcia S, Rocheford TR, McMullen MD, Holland JB, Buckler ES. Genome-wide association study of leaf architecture in the maize nested association mapping population. Nat Genet. 2011;43:159-62.

2. Elshire RJ, Glaubitz JC, Sun Q, Poland JA, Kawamoto K, Buckler ES, Mitchell SE. A robust, simple genotyping-by-sequencing (GBS) approach for high diversity species. PLoS One. 2011;6(5):e19379.

3. Ganal MW, Durstewitz G, Polley A, Bérard A, Buckler ES, Charcosset A, Clarke JD, Graner E-M, Hansen M, Joets J, et al. A Large Maize (Zea mays L.) SNP Genotyping Array: Development and Germplasm Genotyping, and Genetic Mapping to Compare with the B73 Reference Genome. PLoS ONE. 2011; 6(11):e28334.

4. Unterseer S, Bauer E, Haberer G, Seidel M, Knaak C, Ouzunova M, Meitinger T, Strom TM, Fries R, Pausch $H$, et al. A powerful tool for genome analysis in maize: development and evaluation of the high density $600 \mathrm{k}$ SNP genotyping array. BMC Genomics. 2014;15(1):823.

5. Zhou X, Stephens M. Genome-wide efficient mixed-model analysis for association studies. Nat Genet. 2012;44:821.

6. Lippert C, Listgarten J, Liu Y, Kadie CM, Davidson RI, Heckerman D. FaST linear mixed models for genome-wide association studies. Nat Methods. 2011;8:833.

7. Listgarten J, Lippert C, Kadie CM, Davidson Rl, Eskin E, Heckerman D. Improved linear mixed models for genome-wide association studies. Nat Methods. 2012;9:525.

8. Kang HM, Sul JH, Service SK, Zaitlen NA, Kong S-Y, Freimer NB, Sabatti C, Eskin E. Variance component model to account for sample structure in genome-wide association studies. Nat Genet. 2010;42:348.

9. Flint-Garcia SA, Thornsberry JM, Buckler ES. Structure of linkage disequilibrium in plants. Annu Rev Plant Biol. 2003;54(1):357-74.

10. Remington DL, Thornsberry JM, Matsuoka Y, Wilson LM, Whitt SR, Doebley J, Kresovich S, Goodman MM, Buckler ES. Structure of linkage disequilibrium and phenotypic associations in the maize genome. Proc Natl Acad Sci. 2001;98(20):11479.

11. Rincent R, Nicolas S, Bouchet S, Altmann T, Brunel D, Revilla P, Malvar RA, Moreno-Gonzalez J, Campo L, Melchinger AE, et al. Dent and Flint maize diversity panels reveal important genetic potential for increasing biomass production. Theor Appl Genet. 2014;127(11):2313-31.

12. Yu J, Pressoir G, Briggs WH, Vroh Bi I, Yamasaki M, Doebley JF, McMullen MD, Gaut BS, Nielsen DM, Holland JB, et al. A unified mixed-model method for association mapping that accounts for multiple levels of relatedness. Nat Genet. 2006;38:203-8.

13. Rincent R, Moreau L, Monod H, Kuhn E, Melchinger AE, Malvar RA, MorenoGonzalez J, Nicolas S, Madur D, Combes V, et al. Recovering power in association mapping panels with variable levels of linkage disequilibrium. Genetics. 2014;197(1):375-87.

14. Van Inghelandt D, Melchinger AE, Lebreton C, Stich B. Population structure and genetic diversity in a commercial maize breeding program assessed with SSR and SNP markers. Theor Appl Genet. 2010;120(7):1289-99.

15. Nicolas SD, Péros J-P, Lacombe T, Launay A, Le Paslier M-C, Bérard A, Mangin B, Valière S, Martins F, Le Cunff $L$, et al. Genetic diversity, linkage disequilibrium and power of a large grapevine (Vitis vinifera $L$ ) diversity panel newly designed for association studies. BMC Plant Biol. 2016;16(1):74

16. Mangin B, Siberchicot A, Nicolas S, Doligez A, This P, Cierco-Ayrolles C. Novel measures of linkage disequilibrium that correct the bias due to population structure and relatedness. Heredity. 2012;108:285-91.

17. Alexander DH, Novembre J, Lange K. Fast model-based estimation of ancestry in unrelated individuals. Genome Res. 2009;19(9):1655-64.

18. Astle W, Balding DJ. Population structure and cryptic relatedness in genetic association studies. Stat Sci. 2009;24(4):451-71.

19. Pritchard JK, Stephens M, Rosenberg NA, Donnelly P. Association mapping in structured populations. Am J Hum Genet. 2000;67(1):170-81.
20. VanRaden PM. Efficient methods to compute genomic predictions. J Dairy Sci. 2008;91(11):4414-23.

21. Bernardo R. Genomewide markers for controlling background variation in association mapping. Plant Genome. 2013;6.

22. Thornsberry JM, Goodman MM, Doebley J, Kresovich S, Nielsen D, Buckler IV ES. Dwarf8 polymorphisms associate with variation in flowering time. Nat Genet. 2001;28:286.

23. Hufford MB, Lubinksy P, Pyhäjärvi T, Devengenzo MT, Ellstrand NC, RossIbarra J. The genomic signature of crop-wild introgression in maize. PLoS Genet. 2013;9(5):e1003477.

24. Rincent $R$, Laloë D, Nicolas $S$, Altmann T, Brunel D, Revilla P, Rodríguez VM, Moreno-Gonzalez J, Melchinger A, Bauer E, et al. Maximizing the Reliability of Genomic Selection by Optimizing the Calibration Set of Reference Individuals: Comparison of Methods in Two Diverse Groups of Maize Inbreds (Zea mays L.). Genetics. 2012;192(2):715.

25. Bouchet S, Bertin P, Presterl T, Jamin P, Coubriche D, Gouesnard B, Laborde $\mathrm{J}$, Charcosset A. Association mapping for phenology and plant architecture in maize shows higher power for developmental traits compared with growth influenced traits. Heredity. 2017;118:249-59.

26. Bouchet $S$, Servin B, Bertin P, Madur D, Combes V, Dumas F, Brunel D, Laborde J, Charcosset A, Nicolas S. Adaptation of maize to temperate climates: mid-density genome-wide association genetics and diversity patterns reveal key genomic regions, with a major contribution of the Vgt2 (ZCN8) locus. PLoS One. 2013;8(8):e71377.

27. Messing J, Dooner HK. Organization and variability of the maize genome. Curr Opin Plant Biol. 2006;9(2):157-63.

28. Hu H, Schrag TA, Peis R, Unterseer S, Schipprack W, Chen S, Lai J, Yan J, Prasanna BM, Nair SK, et al. The genetic basis of haploid induction in maize identified with a novel genome-wide association method. Genetics. 2016;202(4):1267-76.

29. Millet EJ, Welcker C, Kruijer W, Negro S, Coupel-Ledru A, Nicolas SD, Laborde J, Bauland C, Praud S, Ranc N, et al. Genome-wide analysis of yield in Europe: allelic effects vary with drought and heat scenarios. Plant Physiol. 2016;172(2):749-64.

30. Crossa J, Beyene Y, Kassa S, Pérez P, Hickey JM, Chen C, de los Campos G, Burgueño J, Windhausen VS, Buckler E, et al. Genomic Prediction in Maize Breeding Populations with Genotyping-by-Sequencing. G3: Genes|Genomes|Genetics. 2013;3(11):1903-26.

31. Romay MC, Millard MJ, Glaubitz JC, Peiffer JA, Swarts KL, Casstevens TM, Elshire RJ, Acharya CB, Mitchell SE, Flint-Garcia SA, et al. Comprehensive genotyping of the USA national maize inbred seed bank. Genome Biol. 2013;14(6):R55.

32. Gouesnard B, Negro S, Laffray A, Glaubitz J, Melchinger A, Revilla P, MorenoGonzalez J, Madur D, Combes V, Tollon-Cordet C, et al. Genotyping-bysequencing highlights original diversity patterns within a European collection of 1191 maize flint lines, as compared to the maize USDA genebank. Theor Appl Genet. 2017;130(10):2165-89.

33. Elbasyoni IS, Lorenz AJ, Guttieri M, Frels K, Baenziger PS, Poland J, Akhunov E. A comparison between genotyping-by-sequencing and array-based scoring of SNPS for genomic prediction accuracy in winter wheat. Plant Sci. 2018;270:123-30.

34. Liu H, Luo X, Niu L, Xiao Y, Chen L, Liu J, Wang X, Jin M, Li W, Zhang Q, et al. Distant eQTLs and non-coding sequences play critical roles in regulating gene expression and quantitative trait variation in maize. Mol Plant. 2017; 10(3):414-26.

35. Torkamaneh D, Belzile F. Scanning and filling: ultra-dense SNP genotyping combining genotyping-by-sequencing, SNP Array and whole-genome resequencing data. PLoS One. 2015;10(7):e0131533.

36. Frascaroli $E$, Schrag TA, Melchinger AE. Genetic diversity analysis of elite European maize (Zea mays L.) inbred lines using AFLP, SSR, and SNP markers reveals ascertainment bias for a subset of SNPS. Theor Appl Genet. 2013;126(1):133-41.

37. Glaubitz JC, Casstevens TM, Lu F, Harriman J, Elshire RJ, Sun Q, Buckler ES. TASSEL-GBS: a high capacity genotyping by sequencing analysis pipeline. PLoS One. 2014;9(2):e90346.

38. Swarts K, Li H, Romero Navarro JA, An D, Romay MC, Hearne S, Acharya C, Glaubitz JC, Mitchell S, Elshire RJ, et al. Novel Methods to Optimize Genotypic Imputation for Low-Coverage, Next-Generation Sequence Data in Crop Plants. The Plant Genome. 2014;7(3).

39. Hill WG, Weir BS. Variances and covariances of squared linkage disequilibria in finite populations. Theor Popul Biol. 1988;33(1):54-78.

40. Charlesworth $\mathrm{D}$, Willis $\mathrm{JH}$. The genetics of inbreeding depression. Nat Rev Genet. 2009;10:783-96. 
41. Hudson RR, Kaplan NL. Deleterious background selection with recombination. Genetics. 1995;141(4):1605-17.

42. Giraud H, Bauland C, Falque M, Madur D, Combes V, Jamin P, Monteil C, Laborde J, Palaffre C, Gaillard A, et al. Reciprocal genetics: identifying QTL for general and specific combining abilities in hybrids between multiparental populations from two maize (Zea mays L.) heterotic groups. Genetics. 2017;207(3):1167-80.

43. Bauer E, Falque M, Walter H, Bauland C, Camisan C, Campo L, Meyer N, Ranc $\mathrm{N}$, Rincent R, Schipprack W, et al. Intraspecific variation of recombination rate in maize. Genome Biol. 2013;14(9):R103.

44. Le Gouis J, Bordes J, Ravel C, Heumez E, Faure S, Praud S, Galic N, Remoué C, Balfourier F, Allard V, et al. Genome-wide association analysis to identify chromosomal regions determining components of earliness in wheat. Theor Appl Genet. 2012;124(3):597-611.

45. Cormier F, Le Gouis J, Dubreuil P, Lafarge S, Praud S. A genome-wide identification of chromosomal regions determining nitrogen use efficiency components in wheat (Triticum aestivum L). Theor Appl Genet. 2014;127(12):2679-93.

46. Springer NM, Ying K, Fu Y, Ji T, Yeh C-T, Jia Y, Wu W, Richmond T, Kitzman J, Rosenbaum $\mathrm{H}$, et al. Maize Inbreds exhibit high levels of copy number variation (CNV) and presence/absence variation (PAV) in genome content. PLoS Genet. 2009;5(11):e1000734.

47. Darvasi A, Weinreb A, Minke V, Weller Jl, Soller M. Detecting marker-QTL linkage and estimating QTL gene effect and map location using a saturated genetic map. Genetics. 1993;134(3):943-51.

48. Gabriel SB, Schaffner SF, Nguyen H, Moore JM, Roy J, Blumenstiel B, Higgins J, DeFelice M, Lochner A, Faggart M, et al. The structure of haplotype blocks in the human genome. Science. 2002;296(5576):2225-9.

49. Wang H, Chu WS, Hemphill C, Elbein SC. Human Resistin gene: molecular scanning and evaluation of association with insulin sensitivity and type 2 diabetes in Caucasians. J Clin Endocrinol Metab. 2002;87(6):2520-4.

50. Liang Y, Liu Q, Wang X, Huang C, Xu G, Hey S, Lin H-Y, Li C, Xu D, Wu L, et al. ZmMADS69 functions as a flowering activator through the ZmRap2.7ZCN8 regulatory module and contributes to maize flowering time adaptation. New Phytol. 2019;221(4):2335-47.

51. Larièpe A, Mangin B, Jasson S, Combes V, Dumas F, Jamin P, Lariagon C, Jolivot D, Madur D, Fiévet J, et al. The genetic basis of heterosis: multiparental quantitative trait loci mapping reveals contrasted levels of apparent overdominance among traits of agronomical interest in Maize (\&lt; em\&gt;Zea mays\&lt;/em\&gt; L.). Genetics. 2012;190(2):795-811.

52. Butler DG, Cullis BR, Gilmour AR, Gogel BJ. ASReml-R reference manual. Brisbane: The State of Queensland, Department of Primary Industries and Fisheries; 2009.

53. Schnable PS, Ware D, Fulton RS, Stein JC, Wei F, Pasternak S, Liang C, Zhang J, Fulton L, Graves TA, et al. The B73 maize genome: complexity, diversity, and dynamics. Science. 2009;326(5956):1112-5.

54. Ganal MW, Altmann T, Röder MS. SNP identification in crop plants. Curr Opin Plant Biol. 2009;12(2):211-7.

55. Gore MA, Chia J-M, Elshire RJ, Sun Q, Ersoz ES, Hurwitz BL, Peiffer JA, McMullen MD, Grills GS, Ross-Ibarra J, et al. A first-generation haplotype map of maize. Science. 2009;326(5956):1115-7.

56. Browning SR, Browning BL. Rapid and accurate haplotype phasing and missing-data inference for whole-genome association studies by use of localized haplotype clustering. Am J Hum Genet. 2007;81 (5):1084-97.

57. Nei M. Estimation of average heterozygosity and genetic distance from a small number of individuals. Genetics. 1978;89(3):583-90.

58. Gower JC. Some distance properties of latent root and vector methods used in multivariate analysis. Biometrika. 1966;53(3-4):325-38.

59. Hill WG, Robertson A. Linkage disequilibrium in finite populations. Theor Appl Genet. 1968;38(6):226-31.

60. R Core Team. R: A language and environment for statistical computing. Vienna: R Foundation for Statistical Computing; 2015. https:/www.r-project.org/

61. Moskvina V, Schmidt KM. On multiple-testing correction in genome-wide association studies. Genet Epidemiol. 2008;32(6):567-73.

62. Gao X, Becker LC, Becker DM, Starmer JD, Province MA. Avoiding the high Bonferroni penalty in genome-wide association studies. Genet Epidemiol. 2010;34(1):100-5.

63. Gao X, Starmer J, Martin ER. A multiple testing correction method for genetic association studies using correlated single nucleotide polymorphisms. Genet Epidemiol. 2008;32(4):361-9.

\section{Publisher's Note}

Springer Nature remains neutral with regard to jurisdictional claims in published maps and institutional affiliations.

\section{Ready to submit your research? Choose BMC and benefit from}

- fast, convenient online submission

- thorough peer review by experienced researchers in your field

- rapid publication on acceptance

- support for research data, including large and complex data types

- gold Open Access which fosters wider collaboration and increased citations

- maximum visibility for your research: over $100 \mathrm{M}$ website views per year

At BMC, research is always in progress.

Learn more biomedcentral.com/submissions 